\title{
SINGULAR INTEGRAL OPERATORS ON TENT SPACES
}

\author{
PASCAL AUSCHER, CHRISTOPH KRIEGLER, SYLVIE MONNIAUX, PIERRE PORTAL
}

\begin{abstract}
We extend the recent results concerning boundedness of the maximal regularity operator on tent spaces. This leads us to develop a singular integral operator theory on tent spaces. Such operators have operator-valued kernels. A seemingly appropriate condition on the kernel is time-space decay measured by off-diagonal estimates with various exponents.
\end{abstract}

\section{INTRODUCTION}

Let $-L$ be a densely defined closed linear operator acting on $L^{2}\left(\mathbb{R}^{n}\right)$ and generating a bounded analytic semigroup $\left(e^{-t L}\right)_{t \geq 0}$. Consider the maximal regularity operator originally defined for $f \in L^{2}\left(\mathbb{R}_{+}, d t ; D(L)\right), \mathbb{R}_{+}=(0,+\infty)$, by the Bochner integral

$$
\mathcal{M}_{L} f(t)=\int_{0}^{t} L e^{-(t-s) L} f(s) d s .
$$

This is an example of a singular integral operator with operator-valued kernel. The bounded extension of this operator to $L^{2}\left(\mathbb{R}_{+}, d t ; L^{2}\left(\mathbb{R}^{n}\right)\right)=L^{2}\left(\mathbb{R}_{+}^{n+1}, d t d x\right), \mathbb{R}_{+}^{n+1}=\mathbb{R}_{+} \times \mathbb{R}^{n}$, was established by de Simon in 26. The maximal regularity operator plays a crucial role in evolution equations, where its boundedness is used to deduce a priori estimates, which, in turn, can be used to solve non-autonomous and/or non linear problems (see the lecture notes [21]). It has thus been the source of intense study, especially in the past 10 years, in $L^{p}$, and in Besov spaces. As part of the recent development of an evolution equation approach to boundary value problems on the upper half-space with data in $L^{2}\left(\mathbb{R}^{n}\right)$, based on the functional calculus of appropriate Dirac operators, a weighted version of de Simon's theorem is proven in [3] and [4, Theorem 1.3], but can be essentially attributed to the earlier work [15] (see below).

Theorem 1.1. With $L$ as above, $\mathcal{M}_{L}$ extends to a bounded operator on $L^{2}\left(\mathbb{R}_{+}^{n+1}, t^{\beta} d t d x\right)$ for all $\beta \in(-\infty, 1)$.

This was proven before in 25] for $\beta \in[0,1)$ and a more general class of operators akin to the ones we introduce next, and then for $\beta \in(-1,1)$ in [15, Theorem 1.13] when $L$ has dense range. The range of $\beta$ is shown in 3 to be optimal. Values such as $\beta=-1$ and also an endpoint result for $\beta=1$ were central for applications to the boundary value problems in $[3$. It should be noted, however, that while the statement of [15. Theorem 1.13] does not include the case $\beta=-1$, its proof via their Proposition 1.14 actually gives Theorem 1.1 .

The articles [15] and [25] actually prove weighted $L^{p}$ estimates for $1<p<\infty$ and show that weighted maximal regularity is equivalent to the unweighted one. However, the $L^{p}$ analogue of Theorem 1.1 needed in the applications we have in mind does not involve weighted $L^{p}\left(\mathbb{R}_{+}^{n+1}\right)$ spaces for $p \neq 2$, but more appropriate spaces of functions on the upper half space $\mathbb{R}_{+}^{n+1}$. Let us explain this fact.

Traditionally, an evolution problem of the form $u_{t}+L u=g$, with initial value $u_{0}=$ $f \in L^{p}\left(\mathbb{R}^{n}\right)$, is seen as an ordinary differential equation for $L^{p}\left(\mathbb{R}^{n}\right)$-valued functions. One

Date: revised, March 15, 2012.

1991 Mathematics Subject Classification. 47D06, 47A60, 35K22, 42B35, 42B20.

Key words and phrases. maximal regularity, tent spaces, singular integral operators, off-diagonal estimates.

The second author acknowledges financial support from the Karlsruhe House of Young Scientists (KHYS). 
assumes that $-L$ generates an analytic semigroup on $L^{p}\left(\mathbb{R}^{n}\right)$, and looks for maximal regularity in spaces such as $L^{p}\left(\mathbb{R}_{+} ; L^{p}\left(\mathbb{R}^{n}\right)\right)$. However, if $L=-\operatorname{div} A \nabla$ is a second order, divergence form elliptic operator on $\mathbb{R}^{n}$ with bounded measurable complex valued coefficients, $-L$ only generates an analytic semigroup on $L^{p}\left(\mathbb{R}^{n}\right)$ for $p$ in an interval $\left(p_{-}(L), p_{+}(L)\right)$ including 2 , but not always equal to $(1, \infty)$ (see [1]). In this range, maximal regularity results can be proven using the extrapolation method pioneered by Blunck and Kunstmann in [12, and developed in [1. Outside of that range, however, maximal regularity in $L^{p}\left(\mathbb{R}_{+}^{n+1}\right)$ spaces, weighted or not, cannot hold. In this paper, we prove maximal regularity results on the (unweighted) tent space $T^{p, 2,2}$ for all $p \in\left(\frac{n}{n+1}, \infty\right.$ ] (see Proposition 1.6 below), even though, for small $p,-L$ does not even generate a $C_{0}$-semigroup on $L^{p}\left(\mathbb{R}^{n}\right)$.

Moreover, even when $L=-\Delta$, the free evolution $(t, y) \mapsto e^{t \Delta} f(y)$ does not belong to $L^{p}\left(\mathbb{R}_{+}^{n+1}\right)$ when $f \in L^{p}\left(\mathbb{R}^{n}\right)$. This can be compensated by assuming more regularity on $f$, or by using a weighted $L^{p}\left(\mathbb{R}_{+}^{n+1}\right)$ space with an appropriate weight. However, when dealing with $L^{p}$ initial data (in boundary value problems, or evolution problems with rough data, for instance), it is desirable to use a norm of the heat extension $(t, y) \mapsto e^{t \Delta} f(y)$ that is equivalent to the $L^{p}$ norm of $f$ for $p \in(1, \infty)$, and to its $H^{p}$ norm for $p \in(0,1]$. Weighted $L^{p}\left(\mathbb{R}_{+}^{n+1}\right)$ norms do not have this property, but classical harmonic analysis gives many different norms that do.

The one which is of interest to us is given by the following area integral:

$$
\|f\|_{p} \approx\left(\iint_{\mathbb{R}^{n}}\left(\iint_{\mathbb{R}_{+}^{n+1}} \frac{1}{B\left(x, t^{\frac{1}{2}}\right)(y)} t^{\frac{n}{2}}\left|\Delta e^{t \Delta} f(y)\right|^{2} t d t d y\right)^{\frac{p}{2}} d x\right)^{\frac{1}{p}} .
$$

Such a characterisation of the $L^{p}$ (or $\left.H^{p}\right)$ norm of a function in terms of its heat extension originates from the work of Fefferman-Stein [14. In more recent terminology, this says that $\Delta e^{t \Delta} f$ belongs to a parabolic version of one of the tent spaces introduced by Coifman-MeyerStein 13 .

Now, if one considers the "mild solution" $u$ of $u_{t}-\Delta u=g$ and $u_{0}=0$, given formally by the integral formula $\int_{0}^{t} e^{(t-s) \Delta} g(s) d s$, one is led to consider the boundedness of the maximal regularity operator $\stackrel{0}{\mathcal{M}}_{-\Delta}$ in the norm above. Having such a priori estimates in the same space as the free evolution $(t, y) \mapsto e^{t \Delta} f(y)$ is a first step towards solving, for example, non-linear problems with $L^{p}$ data. Remark that this solution space has, a priori, nothing to do with the space of continuous functions of $t$ with values in $L^{p}$. We thus depart from the tradition of looking at evolution problems for functions on $\mathbb{R}_{+}^{n+1}$ as Banach space valued ODE, and work on spaces where the time and space variables are intrinsically linked. We refer to [10, and the forthcoming 9], for more on the PDE aspect of such questions via a tent space approach. We just mention here that this idea goes back (at least) to Koch-Tataru's work on Navier-Stokes equations [20].

We introduce the alluded variants of the tent spaces as follows. For $0<p<\infty, m \in \mathbb{N}^{*}$, $\beta \in \mathbb{R}$, define the tent space $T^{p, 2, m}\left(t^{\beta} d t d y\right)$ as the space of all locally square integrable functions on $\mathbb{R}_{+}^{n+1}$ such that

$$
\|g\|_{T^{p, 2, m}\left(t^{\beta} d t d y\right)}=\left(\iint_{\mathbb{R}^{n}}\left(\iint_{\mathbb{R}_{+}^{n+1}} \frac{1 B\left(x, t^{\frac{1}{m}}\right)(y)}{t^{\frac{n}{m}}}|g(t, y)|^{2} t^{\beta} d t d y\right)^{\frac{p}{2}} d x\right)^{\frac{1}{p}}<\infty .
$$

The classical case is $\beta=-1, m=1$, in which case, the space is simply denoted by $T^{p, 2}$. Since $\|g\|_{T^{p, 2, m}\left(t^{\beta} d t d y\right)}=\|\tilde{g}\|_{T^{p, 2}}$, where $\tilde{g}(s, y)=\sqrt{m} g\left(s^{m}, y\right) s^{\frac{m(\beta+1)}{2}}, T^{p, 2, m}\left(t^{\beta} d t d y\right)$ is isometric to $T^{p, 2}$. However, the parameter $m$ is needed to handle different homogeneities (corresponding to differential operators of different orders), and the parameter $\beta$ is used to handle different applications (e.g. different degree of smoothness for initial data in evolution problems). We also remark that a simple use of Fubini's theorem shows that $\|g\|_{T^{2,2, m}\left(t^{\beta} d t d y\right)}^{2}=b_{n}\|g\|_{L^{2}\left(\mathbb{R}_{+}^{n+1}, t^{\beta} d t d y\right)}^{2}$, whatever the parameter $m$ is, with $b_{n}$ being the volume of the Euclidean unit ball. Therefore, 
for $p=2$, tent spaces agree with weighted $L^{2}$ spaces. But it is easy to show that it is not true when $p \neq 2$.

The nature of the norm (a quasi-norm when $p<1$ ), makes local square integrability a requirement. As already showed in 7. (and subsequently in 19]) for different types of operators, a pertinent notion for boundedness of the maximal regularity operator on tent spaces is a measure of decay of the semigroup called $\left(L^{2}-L^{2}\right)$ off-diagonal estimates.

Definition 1.2. A family of bounded linear operators $\left(T_{t}\right)_{t \geq 0} \subset B\left(L^{2}\left(\mathbb{R}^{n}\right)\right)$ is said to satisfy off-diagonal estimates of order $M$, with homogeneity $m \in \mathbb{N}^{*}$, if, for all Borel sets $E, F \subset \mathbb{R}^{n}$, all $t>0$, and all $f \in L^{2}\left(\mathbb{R}^{n}\right)$ :

$$
\left\|1_{F} T_{t} 1_{E} f\right\|_{2} \lesssim\left(1+\frac{\operatorname{dist}(E, F)^{m}}{t}\right)^{-M}\left\|1_{E} f\right\|_{2}
$$

Here, and in what follows, $\|\cdot\|_{2}$ denotes the norm in $L^{2}\left(\mathbb{R}^{n}\right)$.

This property is a replacement for pointwise kernel estimates, which is satisfied, for instance, by heat semigroups generated by elliptic operators with rough coefficients. Note that we allow a polynomial decay.

With the definition above, the following result was proved in 8 .

Theorem 1.3. Let $m \in \mathbb{N}^{*}, \beta \in(-\infty, 1), p \in\left(\frac{2 n}{n+m(1-\beta)}, \infty\right) \cap(1, \infty)$, and $\tau=\min (p, 2)$. If $\left(t L e^{-t L}\right)_{t \geq 0}$ satisfies off-diagonal estimates of order $M>\frac{n}{m \tau}$, with homogeneity $m$, then $\mathcal{M}_{L}$ extends to a bounded operator on $T^{p, 2, m}\left(t^{\beta} d t d y\right)$.

The surprise is to obtain results for $p<2$. This is particularly true in applications to stochastic parabolic PDEs. Results in this context have been developed in parallel to this article in [10, which contains lighter versions of some of the material presented here. In the present paper we concentrate more on the abstract theory, and try to weaken assumptions as much as possible. This is important even for maximal regularity operators, see Section 5

An end-point result, for $p=\infty$, was also obtained in [8]. In this context, the appropriate tent space consists of functions such that $|g(t, y)|^{2} \frac{d t d y}{t}$ is a Carleson measure, and is defined as the space of all locally square integrable functions such that

$$
\|g\|_{T^{\infty, 2}}^{2}=\sup _{(x, r) \in \mathbb{R}^{n} \times \mathbb{R}_{+}} r^{-n} \int_{B(x, r)} \int_{0}^{r}|g(t, y)|^{2} \frac{d t d y}{t}<\infty .
$$

The weighted version (defined through a change of variable as above) is given by

$$
\|g\|_{T^{\infty, 2, m}\left(t^{\beta} d t d y\right)}^{2}:=\sup _{(x, r) \in \mathbb{R}^{n} \times \mathbb{R}_{+}} r^{-n} \int_{B(x, r)} \int_{0}^{r^{m}}|g(t, y)|^{2} t^{\beta} d t d y .
$$

Theorem 1.4. Let $m \in \mathbb{N}^{*}$ and $\beta \in(-\infty, 1)$. If $\left(t L e^{-t L}\right)_{t \geq 0}$ satisfies off-diagonal estimates of order $M>\frac{n}{2 m}$, with homogeneity $m$, then $\mathcal{M}_{L}$ extends to a bounded operator on $T^{\infty, 2, m}\left(t^{\beta} d t d y\right)$.

Note that the backward maximal regularity operator

$$
\mathcal{M}_{L}^{-} f(t)=\int_{t}^{\infty} L e^{-(s-t) L} f(s) d s
$$

can be studied on tent spaces, either by duality as $\mathcal{M}_{L}^{-}=\left(\mathcal{M}_{L^{*}}\right)^{*}$, or directly.

Here, we continue the development of such tent space boundedness results, and we obtain three-fold improvements. The main statements are in the core of the article. We give here our motivation and extract sample new results as illustrations.

The first observation is that the conclusion of Theorem 1.3 is far from optimal in concrete situations. For instance, for $-\Delta$ (heat semigroup), and its square root $\sqrt{-\Delta}$ (Poisson semigroup), or even $-\Delta+V$ with $V \in L_{l o c}^{1}\left(\mathbb{R}^{n}\right), V \geq 0$, and its square root, or $-\operatorname{div} A \nabla$ a 
second order divergence form elliptic operator on $\mathbb{R}^{n}$ with bounded, measurable, real-valued coefficients, and its square root, the range of $p$ can be much improved.

Proposition 1.5. (1) $\mathcal{M}_{-\Delta+V}$ and $\mathcal{M}_{-\operatorname{div} A \nabla}$ (with real-valued coefficient matrix $A$ ) extend to bounded operators on $T^{p, 2,2}(d t d y)$ when $\frac{n}{n+1}<p \leq \infty$.

(2) $\mathcal{M}_{\sqrt{-\Delta+V}}$ and $\mathcal{M}_{\sqrt{-\operatorname{div} A \nabla}}$ (with real-valued coefficient matrix $A$ ) extend to bounded operators on $T^{p, 2,1}\left(t^{-1} d t d y\right)$ when $\frac{n}{n+1}<p \leq \infty$.

The range of $p$ is a consequence of the pointwise decay of the corresponding heat kernels. However, not all semigroups obey pointwise decay. In that case, one can use intermediate conditions between pointwise decay and $L^{2}-L^{2}$ off-diagonal estimates such as $L^{q}-L^{r}$ offdiagonal estimates with $q \leq r$ and $q=2$ or $r=2$ (see Definition 2.4). This information can then be used to quantify the range of $p$ for tent space boundedness. This is the case for $-\operatorname{div} A \nabla$ with complex-valued coefficients. Here, the decay is Gaussian but the range of $q$ or $r$ may be limited as dimension increases.

Proposition 1.6. For a complex-valued coefficient matrix $A, \mathcal{M}_{-\operatorname{div}} A \nabla$ extends to a bounded operator on $T^{p, 2,2}(d t d y)$ when $\frac{1}{2}<p \leq \infty$ if $n=1, \frac{2}{3}<p \leq \infty$ if $n=2, \frac{6}{7}-\varepsilon<p \leq \infty$ if $n=3$, and $2-\frac{4}{n}-\varepsilon<p \leq \infty$ if $n \geq 4$. The $\varepsilon>0$ depends on the operator but the lower bound is at least $\frac{n}{n+1}$.

These two propositions (see Section 5 for their proofs) follow from general statements (proved in Sections 3 and 4) in which one requires a lower bound on the polynomial decay exponent $M$ of Definition 2.4. Note that this lower bound increases with dimension. As the decay here is exponential, the exponent $M$ can be as large as one wants, and the results apply.

We now consider the case of polynomial decay. This is our second point. In this case, the value of $M$ is to be compared with the lower bound in our statements for applicability. For example, one has $M=1$ in the $L^{2}-L^{2}$ off-diagonal estimates with homogeneity $m=1$ for $\sqrt{-\operatorname{div} A \nabla}$ (even for $\sqrt{-\Delta}$ ). Theorem 1.3 requires $M>n / \tau$, but one can take advantage of the fact that the exponent $M$ in the $L^{q}-L^{2}$ off-diagonal estimates grows linearly in $1 / q$ (see Proposition 5.3). However, the range of $q$ may be limited as well, which is the case for $-\operatorname{div} A \nabla$ operators with complex-valued coefficients, and again we may not have a large enough value of $M$.

On the other hand, with no decay at all, the $p=2$ boundedness follows from Theorem 1.1. So it seems reasonable to expect a range of $p$ near 2 depending on $q$ and $M$, when $q \sim 2$ and $M>0$ is small, by some kind of interpolation procedure. We will obtain (see Section 5) a general result in this direction, which gives, for this particular operator, the proposition below.

Proposition 1.7. For a complex-valued coefficient matrix $A, \mathcal{M}_{\sqrt{- \text { div } A \nabla}}$ extends to a bounded operator on $T^{p, 2,1}\left(t^{-1} d t d y\right)$ when $\frac{1}{2}<p \leq \infty$ if $n=1, \frac{2}{3}<p \leq \infty$ if $n=2, \frac{6}{7}-\varepsilon<p \leq \infty$ if $n=3,1-\varepsilon<p \leq \infty$ if $n=4$ and $2-\frac{4}{n}-\varepsilon<p<\frac{2 n-4}{n-4}+\varepsilon^{\prime}$ if $n \geq 5$. The $\varepsilon, \varepsilon^{\prime}>0$ depend on the operator but the lower bound is at least $\frac{n}{n+1}$.

To do this interpolation procedure, we view the maximal regularity operator within a family of operators of the same nature. Thus, and this is the third point, it becomes interesting and convenient to develop an abstract formulation that is not restricted to the maximal regularity operator. We introduce, in the next section, a class of singular integral operators in the context of tent spaces. Sufficient conditions for their boundedness are given in Sections 3 and 4. We remark that, in contrast to the usual $L^{p}$ theory for Calderón-Zygmund operators, no regularity of the kernel is necessary. In a sense, despite the fact that tent spaces, for $1<p<\infty$, can be seen as subspaces of Hilbert-valued $\mathrm{L}^{p}$ spaces (18]), Calderón-Zygmund theory does not seem to be an appropriate machinery to study singular integral operators in this context. We depart from the usual treatment of maximal regularity through a singular integral operator acting on some Banach-valued functions. Here, we start from the "easy" Hilbert $\left(L^{2}\right)$ space theory, and then move on to tent spaces, using the notion of $L^{q}-L^{r}$ off-diagonal decay, which extends the notion of $L^{2}-L^{2}$ off-diagonal decay defined above. 
Remark 1.8. Our results can, nevertheless, be extended to the context of tent spaces of Banach space-valued functions (provided the Banach space $X$ is UMD, and $1<p<\infty$ ). This is done by adapting the arguments of 19 to take advantage of $L^{q}-L^{2}$ (for $q \leq 2$, resp. $L^{2}-L^{q}$ for $q \geq 2$ ), rather than $L^{2}-L^{2}$, off-diagonal estimates, in the same way it is done in this paper. However, the obvious adaptation does not seem to produce optimal relationships between $p, q, M$, and the geometry of $X$. We choose not to attempt to address this issue here.

\section{Singular integral operators}

2.1. Abstract setup. Consider a separable complex Hilbert space $H$. For $\beta \in \mathbb{R}$, set $\mathcal{H}_{\beta}=$ $L^{2}\left(\mathbb{R}_{+}, t^{\beta} d t ; H\right)$. We consider the following classes of operators $S I O^{ \pm} \subset \mathcal{B}\left(\mathcal{H}_{0}\right)$.

Definition 2.1. (1) We say $T \in S I O^{+}$if $T \in \mathcal{B}\left(\mathcal{H}_{0}\right)$ and there exist a strongly measurable family of operators $K(t, s) \in \mathcal{B}(H), t, s \in \mathbb{R}_{+}$and a constant $C<\infty$ such that $\|K(t, s)\| \leq C|t-s|^{-1}$ and

$$
T f(t)=\int_{0}^{t} K(t, s) f(s) d s
$$

for all $f \in \mathcal{H}_{0}$ with bounded support in $\mathbb{R}_{+}$and almost all $t \in \mathbb{R}_{+}$not in the support of $f$.

(2) We say $T \in S I O^{-}$if $T \in \mathcal{B}\left(\mathcal{H}_{0}\right)$ and there exist a strongly measurable family of operators $K(t, s) \in \mathcal{B}(H), t, s \in \mathbb{R}_{+}$and a constant $C<\infty$ such that $\|K(t, s)\| \leq$ $C|t-s|^{-1}$ and $T$ has the representation

$$
T f(t)=\int_{t}^{\infty} K(t, s) f(s) d s
$$

for all $f \in \mathcal{H}_{0}$ with bounded support in $\mathbb{R}_{+}$and almost all $t \in \mathbb{R}_{+}$not in the support of $f$.

We remark that $K(t, s)$ need only be defined on $s<t$ for $T \in S I O^{+}$and on $t<s$ for $T \in S I O^{-}$and the value at $t=s$ is irrelevant. With this precaution, we say that $T \in S I O^{ \pm}$ is associated to the operator-valued kernel $K(t, s)$ and that such kernels belong to the class $S K^{ \pm}$of singular kernels.

Our terminology follows in part that of singular integrals (here with operator-valued kernels) but we assume a sign condition on $s-t$.

Let us make a few remarks.

The representation (2.1) of $T f$ above is a Bochner integral and the equality holds in $H$. It is clearly equivalent to

$$
\langle T f, g\rangle=\iint_{s<t}\langle K(t, s) f(s), g(t)\rangle d s d t
$$

for $f, g \in \mathcal{H}_{0}$ having bounded disjoint support. The inner product on the left is the canonical one in $\mathcal{H}_{0}$, and on the right the canonical one in $H$.

It is clear that $T \in S I O^{+}$if and only if $T^{*} \in S I O^{-}$, with associated kernel $K(s, t)^{*}$. Hence, similar comments apply to (2.2).

The basic examples are of course $\mathcal{M}_{L} \in S I O^{+}$and $\mathcal{M}_{L}^{-} \in S I O^{-}$. For $\mathcal{M}_{L}$ the boundedness on $\mathcal{H}_{0}$ is given by de Simon's theorem. Then the formula (2.3) holds for all $f \in$ $L^{2}\left(\mathbb{R}_{+}, d t ; D(L)\right)$ and all $g \in \mathcal{H}_{0}$ with continuous kernel $K(t, s)=L e^{-(t-s) L}$ on $s<t$. If now, $f, g$ have disjoints supports, one can argue by density of $D(L)$ in $H$. For $\mathcal{M}_{L}^{-}$, we simply use $\mathcal{M}_{L}^{-}=\left(\mathcal{M}_{L^{*}}\right)^{*}$.

There is a natural splitting of operators $T \in S^{+} O^{+}$into an integral part plus a singular part. Let $K$ be the associated kernel. Using that $t-s \sim t$ when $s<t / 2$ and Hardy inequality, 
one has

$$
\int_{0}^{\infty}\left(\int_{0}^{\frac{t}{2}}\|K(t, s)\|\|f(s)\| d s\right)^{2} d t \lesssim \int_{0}^{\infty}\left(\frac{1}{t} \int_{0}^{\frac{t}{2}}\|f(s)\| d s\right)^{2} d t \lesssim\|f\|_{\mathcal{H}_{0}}^{2} .
$$

Hence, the integral part of $T$ is the operator defined for $f \in \mathcal{H}_{0}$ for almost all $t>0$ by the Bochner integral

$$
\left(T_{2} f\right)(t)=\int_{0}^{\frac{t}{2}} K(t, s) f(s) d s
$$

and $T_{2} \in S I O^{+}$as well. The singular part is $T_{1}:=T-T_{2} \in S I O^{+}$, and carries the singularity at $s=t$. Its associated kernel is $K(t, s) 1_{t / 2<s<t}$. Note that, for the integral part, the integral representation is valid without restriction on $f$ and $t$.

For $T \in S I O^{-}$, one has the same splitting with $T_{2} f(t)=\int_{2 t}^{\infty} K(t, s) f(s) d s$ as the integral part, and $T_{1}=T-T_{2}$ as the singular part.

Theorem 1.1 and its proof carry to this abstraction.

Theorem 2.2. Let $\beta \in(-\infty, 1)$. Any operator in $T \in S I O^{+}$extends to a bounded operator on $\mathcal{H}_{\beta}$ which is denoted by $T$ as well. Furthermore, for any kernel $K \in S K^{+}$and $f \in \mathcal{H}_{\beta}, \int_{0}^{\frac{t}{2}}\|K(t, s)\|\|f(s)\| d s$ is an element of $L^{2}\left(\mathbb{R}_{+}, t^{\beta} d t\right)$, so that for almost all $t>0$, $\int_{0}^{\frac{t}{2}} K(t, s) f(s) d s$ is a Bochner integral in $H$. If, in particular, $K$ is the kernel of $T$ then this integral agrees with $\left(T_{2} f\right)(t)$.

The same statement holds for $T \in S I O^{-}$and $-\beta \in(-\infty, 1)$.

We include a quick argument. For $\alpha=\beta / 2<1 / 2$, we have that

$$
\int_{0}^{\infty}\left(\int_{0}^{t}\|K(t, s)\|\left|t^{\alpha}-s^{\alpha}\right| s^{-\alpha}\left\|s^{\alpha} f(s)\right\| d s\right)^{2} d t \lesssim\|f\|_{\mathcal{H}_{\beta}}^{2}
$$

using the Schur test and the bound on $K$. Hence, the integral operator $f \mapsto\left[t \mapsto \int_{0}^{t} K(t, s)\left(t^{\alpha}-\right.\right.$ $\left.\left.s^{\alpha}\right) f(s) d s\right]$ is bounded from $\mathcal{H}_{\beta}$ to $\mathcal{H}_{0}$. For $f \in \mathcal{H}_{\beta}$ with compact support in $\mathbb{R}_{+}$, it agrees with $t^{\alpha}(T f)(t)-\left(T\left(s^{\alpha} f\right)\right)(t)$. Since $T \in B\left(\mathcal{H}_{0}\right)$, this readily gives the result by density.

The second part follows from the weighted Hardy inequalities [22] when $\beta<1$

$$
\int_{0}^{\infty}\left(\frac{1}{t} \int_{0}^{\frac{t}{2}}\|f(s)\| d s\right)^{2} t^{\beta} d t \lesssim\|f\|_{\mathcal{H}_{\beta}}^{2}
$$

The proof for $\mathrm{SIO}^{-}$is left to the reader.

2.2. Concrete situation. Now, in order to get tent space results, we specialise to $H=$ $L^{2}\left(\mathbb{R}^{n}\right)$, and introduce subclasses. First recall that $\mathcal{H}_{\beta}$ can be identified with $L^{2}\left(\mathbb{R}_{+}^{n+1}, t^{\beta} d t d y\right)$. Hence, we now write $f(s)$ as $f$ or $f(s, \cdot)$ if we want to specialise the $s$ variable. Using that we have a spatial variable, we extend (2.1) as follows.

Lemma 2.3. Let $\beta<1$ and $T \in S I O^{+}$. Let $E, F$ be two Borel sets of $\mathbb{R}^{n}$ and $I, J$ two open intervals in $\mathbb{R}_{+}$. Assume that $f \mapsto\left[(t, y) \mapsto \int_{0}^{t}|(K(t, s) f(s, \cdot))(y)| d s\right]$ is bounded from the space of functions $f \in L^{2}\left(s^{\beta} d s d x\right)$ with support in $I \times E$ into $L^{2}\left(J \times F, t^{\beta} d t d y\right)$. Then the representation $T f(t, y)=\int_{0}^{t}(K(t, s) f(s, \cdot))(y) d s$ holds for all such $f$ with equality in $L^{2}(J \times$ $\left.F, t^{\beta} d t d y\right)$. 
The corresponding statement holds for $T \in S I O^{-}$and $-\beta<1$.

Remark that this lemma is only needed for singular parts. For regular parts, the representation is valid without support conditions.

Proof. Both terms are defined in $L^{2}\left(J \times F, t^{\beta} d t d y\right)$ by assumption so that it suffices to prove the following claim:

$$
\langle T f, g\rangle=\iint_{J \times F}\left(\int_{0}^{t}(K(t, s) f(s, \cdot))(y) d s\right) \bar{g}(t, y) d t d y
$$

for all $f \in C_{0}^{\infty}\left(I ; L^{2}(E)\right)$ and $g \in C_{0}^{\infty}\left(J ; L^{2}(F)\right)$. We implicitly extend $f(s, \cdot)$ by 0 outside $E$ and $g(t, \cdot)$ by 0 outside $F$. Remark that, from the assumption, $(s, t, y) \mapsto(K(t, s) f(s, \cdot))(y) \bar{g}(t, y) 1_{s<t}$ is integrable with integral bounded by $\|f\|_{L^{2}\left(s^{\beta} d s d x\right)}\|g\|_{L^{2}\left(t^{\beta} d t d y\right)}$, hence, by Fubini's theorem, we only have to show

$$
\langle T f, g\rangle=\iint_{s<t}\langle K(t, s) f(s, \cdot), g(t, \cdot)\rangle d s d t
$$

Choose orthonormal bases $\left(e_{j}\right)$ of $L^{2}(E)$ and $\left(\varepsilon_{k}\right)$ of $L^{2}(F)$. By a limiting argument for each term, it is enough to assume that $f(s, \cdot)$ and $g(t, \cdot)$ take values in finite dimensional linear spans of the respective bases. Indeed, use boundedness of $T$ in the left hand side and the integrability assumption in the right hand side. By linearity, it is enough to assume that $f(s, \cdot)=f_{j}(s) e_{j}$ and $g(t, \cdot)=g_{k}(t) \varepsilon_{k}$ for scalar test functions $f_{j}, g_{k}$. In this case, there is a distribution $S_{j, k} \in \mathcal{D}^{\prime}(I \times J)$ such that $\langle T f, g\rangle=\left(S_{j, k}(t, s), f_{j}(s) \overline{g_{k}}(t)\right)$. It follows from (2.3) and decomposing on the orthonormal bases that $\left\langle K(t, s) e_{k}, \varepsilon_{j}\right\rangle$ is the restriction to $0<s<t<\infty, s \in I, t \in J$ of $S_{j, k}$. Thus the desired equality holds for such $f, g$ and we are done.

We skip the similar proof for $T \in S \mathrm{IO}^{-}$.

In applications, it suffices to show (absolute) convergence of the integral $\int_{0}^{t} K(t, s) f(s, \cdot) d s$ in the norm $L^{2}\left(J \times F, t^{\beta} d t d y\right)$ to obtain an estimate of $T f$ in that norm, when $f$ is supported in $I \times E$. We shall use this when $E$ and $F$ are at positive distance and $K(t, s)$ satisfies certain decay estimates.

We thus introduce subclasses of $S I O^{ \pm}$, where the size estimate $\|K(t, s)\| \lesssim|t-s|^{-1}$ is complemented by the following time-space estimates.

Definition 2.4. Let $1 \leq q \leq r \leq \infty$. An operator-valued kernel $K=(K(t, s))_{t, s>0} \subset$ $B\left(L^{2}\left(\mathbb{R}^{n}\right)\right)$ is said to satisfy $L^{q}-L^{r}$ decay of order $M>0$, with homogeneity $m \in \mathbb{N}^{*}$, if, for all Borel sets $E, F \subset \mathbb{R}^{n}$, all $t \neq s$, and all $f \in L^{2}\left(\mathbb{R}^{n}\right) \cap L^{q}\left(\mathbb{R}^{n}\right)$ :

$$
\left\|1_{F} K(t, s) 1_{E} f\right\|_{r} \lesssim|t-s|^{-1-\frac{n}{m}\left(\frac{1}{q}-\frac{1}{r}\right)}\left(1+\frac{\operatorname{dist}(E, F)^{m}}{|t-s|}\right)^{-M}\left\|1_{E} f\right\|_{q}
$$

Here, and in what follows $\|\cdot\|_{q}$ denotes the norm in $L^{q}\left(\mathbb{R}^{n}\right)$.

Note that, in the proofs, one only needs this property for sets of the form $E=B(x, r)$ and $F=B\left(x, 2^{k+1} r\right) \backslash B\left(x, 2^{k} r\right)$ (or vice versa). For this restricted property, $L^{q}-L^{r}$ decay implies $L^{\tilde{q}}-L^{\tilde{r}}$ decay for $q \leq \tilde{q} \leq \tilde{r} \leq r$ (by Hölder's inequality), but the order of decay changes. See [6] for more on this issue. We do not, however, use this fact in this paper.

We need only two specific cases: $1 \leq q \leq 2$ and $r=2$, and $q=2$ and $2 \leq r \leq \infty$. In certain cases, the decay is actually exponential, so the polynomial decay defined above holds for all $M>0$, in which case we say that the order is $\infty$. In this paper, we are particularly interested in obtaining results under minimal values of polynomial decay.

Definition 2.5. Let $1 \leq q \leq \infty$ and $M \in \mathbb{R}_{+} \cup\{\infty\}$. We say that $\mathbf{T} \in \mathbf{S I O}_{\mathbf{m}, \mathbf{q}, \mathbf{M}}^{ \pm}$if $T \in S I O^{ \pm}$ and the associated operator-valued kernel $K(t, s) \in S K^{ \pm}$satisfies $L^{q}-L^{2}$ (resp. $L^{2}-L^{q}$ ) decay of order $M$, with homogeneity $m$, when $q \leq 2$ (resp. $q \geq 2$ ). 
The value of $m$ is dictated by the situation, and $q$ and $M$ are the most important parameters. Let us point out that all calculations work with $m$ being any positive real number, rather than just integer. We mention this for potential development towards fractal situations where fractional homogeneity can occur.

\section{ROLE OF $L^{q}-L^{2}$ DECAY}

The range of $p$ below 2 for which $T^{p, 2}$ boundedness results hold can be quantified by $L^{q}-L^{2}$ decay. Some technical conditions are also required. In particular the order $M$ should not be too small.

Theorem 3.1. Let $T \in S I O_{m, q, M}^{+}$with $1 \leq q \leq 2, M>\frac{n}{2 m}$ and let $p_{M}<1$ be defined by $M=\frac{n}{2 m}\left(\frac{2}{p_{M}}-1\right)$. Let $q^{\prime}$ be the dual exponent to $q$ and $\beta<1$.

(1) If $q^{\prime} \leq \frac{2 n}{m(1-\beta)}$ or equivalently $\frac{n}{2 m} \geq-\frac{\beta-1}{2}+\frac{n}{m}\left(\frac{1}{q}-\frac{1}{2}\right)$ then $T$ extends to a bounded operator on $T^{p, 2, m}\left(t^{\beta} d t d y\right)$ when $2 \geq p>p_{c}$, where

$$
p_{c}=\frac{2\left(\frac{n}{2 m}-\frac{n}{m}\left(\frac{1}{q}-\frac{1}{2}\right)\right)}{\frac{n}{2 m}-\frac{n}{m}\left(\frac{1}{q}-\frac{1}{2}\right)+\frac{1-\beta}{2}}=\frac{4 n}{2 n+m(1-\beta) q^{\prime}} \geq 1 .
$$

(2) If $q^{\prime}>\frac{2 n}{m(1-\beta)}$ or equivalently $-\frac{\beta-1}{2}+\frac{n}{m}\left(\frac{1}{q}-\frac{1}{2}\right)>\frac{n}{2 m}$ then $T$ extends to a bounded operator on $T^{p, 2, m}\left(t^{\beta} d t d y\right)$ when $2 \geq p>\sup \left(p_{M}, \tilde{p}_{c}\right)$, where

$$
\tilde{p}_{c}=\frac{2 n}{\frac{2 n}{q}+m(1-\beta)}<1 \text {. }
$$

Let us say a word on the exponents $p_{c}, \tilde{p}_{c}$. In the first case, $p_{c} \geq 1$. In the second case, $\tilde{p}_{c}<1$. It is consistent as

$$
\tilde{p}_{c}=p_{c} \Longleftrightarrow \tilde{p}_{c}=1 \Longleftrightarrow p_{c}=1 \Longleftrightarrow \frac{n}{2 m}=-\frac{\beta-1}{2}+\frac{n}{m}\left(\frac{1}{q}-\frac{1}{2}\right) .
$$

When $q$ is small, we thus get results for $p$ below 1 provided $M$ is not too small (e.g. in the case of exponential decay).

As a function of $q$, the exponents $p_{c}, \tilde{p}_{c}$ are increasing. When $q=2, \tilde{p}_{c}=\frac{2 n}{n+m(1-\beta)}$ which is the exponent found in Theorem [1.3. Remark that we improve over the lower bound: $M>\frac{n}{2 m}$ suffices here instead of $M>\frac{n}{p m}$ when $p \leq 2$.

In [8, Theorem 1.3 was proved using comparison of tent space norms under change of apertures, i.e. $B\left(x, t^{\frac{1}{m}}\right)$ changed to $B\left(x, c t^{\frac{1}{m}}\right)$ for $c>1$. The sharp behavior of these comparisons was obtained in 2 using atomic decompositions and interpolation. It is thus natural to use atoms here as well to prove our results. Furthermore, it simplifies the proofs greatly.

Recall that for $0<p \leq 1$, the tent space $T^{p, 2}$ has an atomic decomposition [13: A $T^{p, 2}$ atom is a function $a(t, y)$ supported $\sqrt{1}$ in a region $(0, r] \times B$ where $B$ is a (closed) ball on $\mathbb{R}^{n}$ of radius $r$, satisfying $\int_{B} \int_{0}^{r}|a(t, y)|^{2} \frac{d t d y}{t} \leq r^{-n\left(\frac{2}{p}-1\right)}$. Any $T^{p, 2}$ function $g$ can be represented as a series $g=\sum \lambda_{j} a_{j}$ where $a_{j}$ is a $T^{p, 2}$ atom and $\sum\left|\lambda_{j}\right|^{p} \sim\|g\|_{T^{p, 2}}^{p}$. Here the series converges in the tent space quasi-norm, and, in particular, in $L_{l o c}^{2}\left(\mathbb{R}_{+}^{n+1}\right)$. Translating this to our setting, $T^{p, 2, m}\left(t^{\beta} d t d y\right)$ atoms are functions $A(t, y)$ with support in $\left(0, r^{m}\right] \times B$, where $B$ is a (closed) ball in $\mathbb{R}^{n}$ of radius $r$, satisfying $\int_{B} \int_{0}^{r^{m}}|A(t, y)|^{2} t^{\beta} d t d y \leq r^{-n\left(\frac{2}{p}-1\right)}$, and the decomposition theorem holds in $T^{p, 2, m}\left(t^{\beta} d t d y\right)$. Remark that atoms are also special elements of $L^{2}\left(\mathbb{R}_{+}^{n+1}, t^{\beta} d t d y\right)=T^{2,2, m}\left(t^{\beta} d t d y\right)$ which is helpful for representation purposes of $S I O^{ \pm}$ operators acting on them.

\footnotetext{
${ }^{1}$ The support is a relatively closed subset of $\mathbb{R}_{+}^{n+1}$.
} 
Remark 3.2. Recall that the map $j: T^{p, 2, m}\left(t^{\beta} d t d y\right) \rightarrow T^{p, 2,1}\left(t^{-1} d t d y\right)$ defined by $j(f)(t, y)=$ $\sqrt{m} t^{\frac{m(1+\beta)}{2}} f\left(t^{m}, y\right)$ is an isometry; it also sends $T^{p, 2, m}\left(t^{\beta} d t d y\right)$ atoms to $T^{p, 2,1}\left(t^{-1} d t d y\right)$ atoms.

Lemma 3.3. Let $p \leq 1$ and $T$ a linear operator bounded on $T^{2,2, m}\left(t^{\beta} d t d y\right)$. Then $T$ has a bounded extension from $T^{p, 2, m}\left(t^{\beta} d t d y\right) \cap T^{2,2, m}\left(t^{\beta} d t d y\right)$ to $T^{p, 2, m}\left(t^{\beta} d t d y\right)$ if it is uniformly bounded on $T^{p, 2, m}\left(t^{\beta} d t d y\right)$ atoms.

Proof. Adapt to $p \leq 1$ the argument in Step 3 of the proof of Theorem 4.9 in [7 done for $T^{p, 2,1}\left(t^{-1} d t d y\right)$ (without loss of generality, one can take $m=1$, and $\beta=-1$ by Remark 3.2). This argument also furnishes the extension procedure.

Theorem 3.1 follows immediately from the two lemmas below applied to the decomposition of $T \in S I O_{m, q, M}^{+}$into its singular part $T_{1}$ plus its integral part $T_{2}$. Recall that $M>\frac{n}{2 m}$.

Lemma 3.4. The operator $T_{1}$ extends to $T^{p, 2, m}\left(t^{\beta} d t d y\right)$ for $p>p_{M}$.

Lemma 3.5. The statement of Theorem 3.1 holds for $T_{2}$.

Proof of Lemma 3.4. By interpolation (see [13] for the case $m=1, \beta=-1$, and apply Remark 3.2 to deduce the general case) it suffices to consider $p_{M}<p \leq 1$. By Lemma 3.3, it is enough to show that $T_{1} A \in T^{p, 2, m}\left(t^{\beta} d t d y\right)$ if $A$ is a $T^{p, 2, m}\left(t^{\beta} d t d y\right)$ atom, with a uniform bound. Since the proofs are scale invariant, we assume that $A$ is supported in $(0,1] \times B(0,1)$. Then we remark that if $t>2, T_{2} A(t, \cdot)=T A(t, \cdot)$ because of the definition of $T_{2}$ and the support of $A$. Hence $\left(T_{1} A\right)(t, \cdot)=0$ for $t>2$. We let $f_{j}(t, y)=\left(T_{1} A\right)(t, y)$ if $2^{j} \leq|y|<2^{j+1}$, 0 elsewhere, and $f_{0}(t, y)=\left(T_{1} A\right)(t, y)$ if $|y| \leq 2,0$ elsewhere. We show that $f_{j}=\lambda_{j} A_{j}$ with $A_{j}$ a $T^{p, 2, m}\left(t^{\beta} d t d y\right)$ atom and $\sum\left|\lambda_{j}\right|^{p} \lesssim 1$.

For $j=0$, this follows from the boundedness of $T_{1}$ on $T^{2,2, m}\left(t^{\beta} d t d y\right)$ as $\beta<1$. For $j \geq 1$, we argue as follows:

$$
\begin{aligned}
\int_{B\left(0,2^{j+1}\right)} \int_{0}^{2^{(j+1) m}} & \left|f_{j}(t, y)\right|^{2} t^{\beta} d t d y \\
& =\int_{0}^{2} \int_{2^{j} \leq|y|<2^{j+1}}\left|\left(T_{1} A\right)(t, y)\right|^{2} d y t^{\beta} d t \\
& =\int_{0}^{2} \int_{2^{j} \leq|y|<2^{j+1}}\left|\int_{\frac{t}{2}}^{t}\left(\frac{t-s}{t-s}\right)^{\varepsilon-\frac{1}{2}}(K(t, s) A(s, \cdot))(y) d s\right|^{2} d y t^{\beta} d t \\
& \lesssim \int_{0}^{2} \int_{2^{j} \leq|y|<2^{j+1}} \int_{\frac{t}{2}}^{t} t^{2 \varepsilon}(t-s)^{1-2 \varepsilon}|(K(t, s) A(s, \cdot))(y)|^{2} d s d y t^{\beta} d t \\
& \lesssim \int_{0}^{2} \int_{\frac{t}{2}}^{t} t^{2 \varepsilon} \frac{1}{(t-s)^{1+2 \varepsilon+\frac{2 n}{m}\left(\frac{1}{q}-\frac{1}{2}\right)}\left(1+\frac{2^{j m}}{t-s}\right)^{-2 M}\|A(s, .)\|_{q}^{2} t^{\beta} d s d t} \\
& \lesssim \int_{0}^{1}\|A(s, .)\|_{2}^{2} s^{\beta} s^{2 \varepsilon} \int_{s}^{2 s} \frac{1}{(t-s)^{1+2 \varepsilon+\frac{2 n}{m}\left(\frac{1}{q}-\frac{1}{2}\right)}}\left(1+\frac{2^{j m}}{t-s}\right)^{-2 M} d t d s \\
& \lesssim 2^{-2 j m M} \int_{0}^{1}\|A(s, .)\|_{2}^{2} s^{\beta} d s .
\end{aligned}
$$

We used Cauchy-Schwarz inequality in the fourth line and $t^{2 \varepsilon} \approx \int_{\frac{t}{2}}^{t}(t-s)^{2 \varepsilon-1} d s$ when $\varepsilon>0$. In the next to last line, we impose $\varepsilon<M-\frac{n}{m}\left(\frac{1}{q}-\frac{1}{2}\right)$, which is possible as $M>\frac{n}{2 m}$ and 
$q \geq 1$. The estimate $\|A(s, .)\|_{q} \lesssim\|A(s, .)\|_{2}$ uses the fact that $A(s, \cdot)$ is supported in $B(0,1)$. As $\gamma=2 m M-n\left(\frac{2}{p}-1\right)>0$, we thus get the desired estimate with $\lambda_{j}=C 2^{-j \gamma / 2}$. We also remark that we implicitly used Lemma 2.3 , which is possible since the last four lines yield the required estimate to write $T_{1} A(t, y)=\int_{\frac{t}{2}}^{t}(K(t, s) A(s, \cdot))(y) d s$ on the support of $f_{j}$.

Proof of Lemma 3.5. We imbed $T_{2}$ into an analytic family of integral operators $\mathcal{J}_{\alpha}$ defined for $\alpha \in \mathbb{C}$ by

$$
\mathcal{J}_{\alpha} f(t, y)=\int_{0}^{\frac{t}{2}}\left(\frac{s}{t}\right)^{\alpha}(K(t, s) f(s, \cdot))(y) d s
$$

Observe that

$$
\iint_{\mathbb{R}_{+}^{n+1}}\left|\mathcal{J}_{\alpha} f(t, y)\right|^{2} t^{\beta} d t d y=\iint_{\mathbb{R}_{+}^{n+1}}\left|\int_{0}^{\frac{t}{2}}\left(\frac{s}{t}\right)^{\alpha-\frac{\beta-1}{2}}\left(t K(t, s)\left(s^{\frac{\beta+1}{2}} f(s, \cdot)\right)\right)(y) \frac{d s}{s}\right|^{2} \frac{d t d y}{t} .
$$

An application of Schur's lemma, using that $t \sim t-s$ and the uniform boundedness of $t K(t, s)$, shows that, provided $\Re e \alpha-\frac{\beta-1}{2}>0$, the last integral is bounded by

$$
C\left(\Re e \alpha-\frac{\beta-1}{2}\right) \iint_{\mathbb{R}_{+}^{n+1}}\left|s^{\frac{\beta+1}{2}} f(s, x)\right|^{2} \frac{d s d x}{s}=C\left(\Re e \alpha-\frac{\beta-1}{2}\right) \iint_{\mathbb{R}_{+}^{n+1}}|f(s, x)|^{2} s^{\beta} d s d x .
$$

Hence, $\mathcal{J}_{\alpha}$ is well-defined for $\Re e \alpha>\frac{\beta-1}{2}$ and bounded on $T^{2,2, m}\left(t^{\beta} d t d y\right)$ for all $m$. Notice that $\beta<1$ implies that this domain contains $\alpha=0$ and $\mathcal{J}_{0}=T_{2}$.

Now we let $A$ be a $T^{p, 2, m}\left(t^{\beta} d t d y\right)$ atom and estimate $\mathcal{J}_{\alpha} A$. Since the proof below is scale invariant, we assume that $A$ is supported in $(0,1] \times B(0,1)$. We let

$$
f_{j}(t, y)=\left\{\begin{array}{l}
\left(\mathcal{J}_{\alpha} A\right)(t, y) \text { if } 2^{j} \leq|y|<2^{j+1} \text { and } t<2^{j m} \\
\left(\mathcal{J}_{\alpha} A\right)(t, y) \text { if }|y|<2^{j+1} \text { and } 2^{j m} \leq t<2^{(j+1) m} \\
0 \text { otherwise, }
\end{array}\right.
$$

for $j \neq 0$ and $f_{0}(t, y)=\left(\mathcal{J}_{\alpha} A\right)(t, y)$ if $|y| \leq 2$ and $t<2^{m}, 0$ elsewhere, so that $\mathcal{J}_{\alpha} A=$ $f_{0}+f_{1}+\ldots$

By the boundedness property of $\mathcal{J}_{\alpha}$, we get

$$
\int_{B(0,2)} \int_{0}^{2^{m}}\left|f_{0}(t, y)\right|^{2} t^{\beta} d t d y \leq C\left(\Re e \alpha-\frac{\beta-1}{2}\right) \int_{B(0,1)} \int_{0}^{1}|A(s, x)|^{2} s^{\beta} d s d x \leq C\left(\Re e \alpha-\frac{\beta-1}{2}\right) .
$$

Next,

$$
\begin{aligned}
\int_{B\left(0,2^{j+1}\right)} & \int_{0}^{2^{(j+1) m}}\left|f_{j}(t, y)\right|^{2} t^{\beta} d t d y= \\
& \int_{2^{j}<|y|<2^{j+1}} \int_{0}^{2^{j m}}\left|f_{j}(t, y)\right|^{2} t^{\beta} d t d y \\
& +\int_{|y|<2^{j+1}} \int_{2^{j m}}^{2^{(j+1) m}}\left|f_{j}(t, y)\right|^{2} t^{\beta} d t d y .
\end{aligned}
$$

Call $I_{j}$ and $J_{j}$ the square roots of the first and second integrals. For $I_{j}$, we split the integral in $s$ defining $\mathcal{J}_{\alpha} A(t, y)$ as

$$
\sum_{k \geq 1} \int_{2^{-k-1} t}^{2^{-k} t}\left(\frac{s}{t}\right)^{\alpha}(K(t, s) A(s, \cdot))(y) d s
$$


so that by Minkowski inequality $I_{j} \leq \sum_{k \geq 1} I_{j, k}$ with

$$
I_{j, k}^{2}=\int_{2^{j}<|y|<2^{j+1}} \int_{0}^{2^{j m}}\left|\int_{2^{-k-1} t}^{2^{-k} t}\left(\frac{s}{t}\right)^{\alpha}(K(t, s) A(s, \cdot))(y) d s\right|^{2} t^{\beta} d t d y .
$$

Using Cauchy-Schwarz inequality in the $s$ integral and then the $L^{q}-L^{2}$ decay with $t \sim t-s$, we get

$$
\begin{aligned}
I_{j, k}^{2} & \lesssim \int_{0}^{2^{j m}} 2^{-k} t \int_{2^{-k-1} t}^{2^{-k} t}\left(\frac{s}{t}\right)^{2 \Re e \alpha} \frac{1}{t^{2+\frac{2 n}{m}\left(\frac{1}{q}-\frac{1}{2}\right)}}\left(1+\frac{2^{j m}}{t}\right)^{-2 M}\|A(s, \cdot)\|_{q}^{2} d s t^{\beta} d t \\
& \lesssim 2^{-2 j m M} \int_{0}^{2^{j m}} 2^{-k} t \int_{2^{-k-1} t}^{2^{-k} t} 2^{-2 k \Re e \alpha} \frac{1}{t^{2+\frac{2 n}{m}\left(\frac{1}{q}-\frac{1}{2}\right)-2 M}}\|A(s, \cdot)\|_{2}^{2} d s t^{\beta} d t \\
& \lesssim 2^{-2 j m M} 2^{k(-2 \Re e \alpha+\beta-1)} \int_{0}^{2^{j m-k}}\|A(s, \cdot)\|_{2}^{2} s^{\beta}\left(2^{k} s\right)^{2 M-\frac{2 n}{m}\left(\frac{1}{q}-\frac{1}{2}\right)} d s .
\end{aligned}
$$

Recall that the support condition on $A$ forces $s \leq 1$. Also $M>\frac{n}{2 m} \geq \frac{n}{m}\left(\frac{1}{q}-\frac{1}{2}\right)$. Using also the size requirement on $A$ we obtain

$$
I_{j, k}^{2} \lesssim 2^{-2 j m M} 2^{k(-2 \Re e \alpha+\beta-1)} 2^{\inf (k, j m)\left(2 M-\frac{2 n}{m}\left(\frac{1}{q}-\frac{1}{2}\right)\right)} .
$$

Hence, $\sum_{k \geq 1} I_{j, k}$ is controlled by $2^{-j m \inf (M, v(\alpha, q))}$ with $v(\alpha, q)=\Re e \alpha-\frac{\beta-1}{2}+\frac{n}{m}\left(\frac{1}{q}-\frac{1}{2}\right)$ if $M \neq v(\alpha, q)$ and by $j m 2^{-j m M}$ if $M=v(\alpha, q)$.

Next, for the second integral, we remark that the support of $A$ forces $s \leq 1$ while $t \sim$ $2^{j m} \geq 2$. Hence

$$
\begin{aligned}
J_{j}^{2} & \leq \int_{|y|<2^{j}} \int_{2^{j m}}^{2^{(j+1) m}} \int_{0}^{1}\left(\frac{s}{t}\right)^{2 \Re e \alpha-(\beta-1)}\left|t\left(K(t, s) s^{\frac{\beta+1}{2}} A(s, \cdot)\right)(y)\right|^{2} \frac{d s}{s} \frac{d t}{t} \\
& \lesssim \int_{2^{j m}}^{2^{(j+1) m}} \int_{0}^{1}\left(\frac{s}{t}\right)^{2 \Re e \alpha-(\beta-1)} \frac{t^{2}}{t^{\frac{2 n}{m}\left(\frac{1}{q}-\frac{1}{2}\right)+2}}\left\|s^{\frac{\beta+1}{2}} A(s, \cdot)\right\|_{q}^{2} \frac{d s}{s} \frac{d t}{t} \\
& \lesssim 2^{-j\left(2\left(\Re e \alpha-\frac{\beta-1}{2}\right)+\frac{2 n}{m}\left(\frac{1}{q}-\frac{1}{2}\right)\right) m}=2^{-2 j m v(\alpha, q)} .
\end{aligned}
$$

We used Hölder's inequality, the size requirement on $A$, and also $s^{2 \Re e \alpha-(\beta-1)} \leq 1$. In all

$$
\left(\int_{|x|<2^{j+1}} \int_{0}^{2^{(j+1) m}}\left|f_{j}(t, y)\right|^{2} t^{\beta} d t d y\right)^{\frac{1}{2}} \lesssim(1+j m) 2^{-j m \inf (M, v(\alpha, q))} .
$$

We now start the discussion. Case (2) corresponds to $v(0, q)>\frac{n}{2 m}$. The exponent $\tilde{p}_{c}$ is such that $v(0, q)=\frac{n}{2 m}\left(\frac{2}{\tilde{p}_{c}}-1\right)$. By Lemma 3.3 . $\mathcal{J}_{0}$ extends to a bounded map on $T^{p, 2, m}\left(t^{\beta} d t d y\right)$ for any $p \leq 1$ with $\frac{n}{2 m}\left(\frac{2}{p}-1\right) \geq \inf (M, v(0, q))$, which means $1 \geq p>\sup \left(p_{M}, \tilde{p}_{c}\right)$. By interpolation with the $p=2$ result, $\mathcal{J}_{0}$ extends to a bounded map on $T^{p, 2, m}\left(t^{\beta} d t d y\right)$ for $\sup \left(p_{M}, \tilde{p}_{c}\right)<p \leq 2$.

Case (1) corresponds to $v(0, q) \leq \frac{n}{2 m}$. Let $\alpha_{1}>0$ be such that $v\left(\alpha_{1}, q\right)=\frac{n}{2 m}$. As in the preceding case, for any $\alpha$ with $\Re e \alpha>\alpha_{1}, \mathcal{J}_{\alpha}$ extends to a bounded map on $T^{1,2, m}\left(t^{\beta} d t d y\right)$ and by checking the proof above, the bound does not depend on $\Im m \alpha$. By the $p=2$ case, if $\alpha_{2}=\frac{\beta-1}{2}<0$, then for any $\alpha$ with $\Re e \alpha>\alpha_{2}, \mathcal{J}_{\alpha}$ extends to a bounded map on $T^{2,2, m}\left(t^{\beta} d t d y\right)$ and the bound does not depend on $\Im m \alpha$. Hence, by Stein's interpolation theorem for analytic families extended to tent spaces (see [18 for its extension to the tent spaces $T^{p, 2}$ with $\left.p \geq 1\right), \mathcal{J}_{0}$ extends to a bounded map on $T^{p, 2, m}\left(t^{\beta} d t d y\right)$ for $p_{c}<p<2$ and 
$p_{c}$ is the exponent with $\frac{1}{p_{c}}=\frac{\theta}{1}+\frac{1-\theta}{2}$ when $0=\theta \alpha_{1}+(1-\theta) \alpha_{2}$. A calculation yields the explicit formula of the statement.

Remark 3.6. Note that the most restrictive conditions on $p$ come from the tail operator $T_{2}$, not the singular one $T_{1}$, which is contrary to usual feeling for singular integral operators. This can be understood by noticing that this tail operator contains the terms where $s$ is close to 0 , and some decay is required to control the tent space norms near this boundary.

We next give a result for operators in $S I O_{m, q, M}^{-}$when $q \leq 2$.

Proposition 3.7. Let $\beta>-1, m \in \mathbb{N}^{*}, T \in S I O_{m, q, M}^{-}$with $1 \leq q \leq 2$ and $M>\frac{n}{2 m}$. Let $p_{M}<1$ be such that $M=\frac{n}{2 m}\left(\frac{2}{p_{M}-1}\right)$. Then $T$ extends to a bounded operator on $T^{p, 2, m}\left(t^{\beta} d t d x\right)$ for $p_{M}<p<2$.

Proof. By interpolation, it suffices to treat the case $p_{M}<p \leq 1$. Take such a $p$. Let $A$ be a $T^{p, 2, m}\left(t^{\beta} d t d y\right)$ atom, i.e. a function supported in some $\left(0, r^{m}\right] \times B\left(x_{0}, r\right)$, and satisfying

$$
\iint_{\mathbb{R}_{+}^{n+1}}|A(s, x)|^{2} s^{\beta} d s d x \leq r^{-n\left(\frac{2}{p}-1\right)} .
$$

For $j \in \mathbb{N}$, let $B_{j}=\left(0,\left(2^{j} r\right)^{m}\right] \times B\left(x_{0}, 2^{j} r\right) \subset \mathbb{R}_{+}^{n+1}$ and $C_{j}=B_{j} \backslash B_{j-1}$ (with $\left.B_{-1}=\emptyset\right)$. For $k, j \in \mathbb{N}$, and $(k, j) \neq(0,0)$ we let

$$
T_{k, j} A(t, y)=1_{C_{j}}(t, y) \int_{2^{k} t}^{2^{k+1} t}(K(t, s) A(s, \cdot))(y) d s
$$

and

$$
\left(T_{0,0} A\right)(t, y)=1_{B_{0}}(t, y)\left(T_{1} A\right)(t, y)
$$

where $T_{1}$ is the singular part of $T$.

We claim that, for a sequence $\lambda_{k, j}>0$, which is independent of $A$ and satisfies $\sum_{k, j=0}^{\infty} \lambda_{k, j}<$ $\infty$, we have

$$
\iint_{B_{j}}\left|T_{k, j} A(t, y)\right|^{2} t^{\beta} d t d y \leq\left(2^{j} r\right)^{-n\left(\frac{2}{p}-1\right)} \lambda_{k, j}^{2},
$$

so $\lambda_{k, j}^{-1} T_{k, j} A$ is a $T^{p, 2, m}\left(t^{\beta} d t d y\right)$ atom. Note that $\sum_{k \geq 1, j \geq 0} T_{k, j} A=T_{2} A$. Using Lemma 2.3 a posteriori, we have $T_{1} A=\sum_{j \geq 0} T_{0, j} A$. Hence $\sum_{k \geq 0, j \geq 0} \bar{T}_{k, j} A=T A$ and thus $\|T A\|_{T^{p, 2, m}\left(t^{\beta} d t d y\right)} \lesssim$ $\sum_{k, j=0}^{\infty} \lambda_{k, j}$. By Lemma 3.3 we are then able to conclude the proof. It remains to prove the claim.

The proof is scale and translation invariant so we assume that $x_{0}=0$ and $r=1$. For $j \geq 1$, we have

$$
\iint_{B_{j}}\left|T_{k, j} A(t, y)\right|^{2} t^{\beta} d t d y \leq \iint_{C_{j}}\left(2^{k} t\right)^{2 \epsilon} \int_{2^{k} t}^{2^{k+1} t}(s-t)^{1-2 \epsilon}|(K(t, s) A(s, \cdot))(y)|^{2} d s t^{\beta} d t d y .
$$

Here we have used the Cauchy-Schwarz inequality as in the proof of Lemma 3.4 and the parameter $\epsilon>0$ will be determined later. Write $C_{j}=\left(0,2^{(j-1) m}\right] \times\left[B\left(0,2^{j}\right) \backslash B\left(0,2^{j-1}\right)\right] \cup$ $\left[2^{(j-1) m}, 2^{j m}\right] \times B\left(0,2^{j}\right)=: C_{j}^{(1)} \cup C_{j}^{(2)}$. If $(t, y) \in C_{j}^{(2)}$, then $t \geq 2^{(j-1) m} \geq 1$, and if $s>$ $2^{k} t \geq 1$, then $A(s, \cdot)=0$. Thus, we can replace $C_{j}$ by $C_{j}^{(1)}$ in the above multiple integral and impose $t \leq 1$. Then we can apply the $L^{q}-L^{2}$ decay with $F=B\left(0,2^{j}\right) \backslash B\left(0,2^{j-1}\right)$ and 
$E=B(0,1)$ to continue estimating as follows

$$
\begin{aligned}
& \leq \int_{0}^{1}\left(2^{k} t\right)^{2 \epsilon} \int_{2^{k} t}^{2^{k+1} t} \frac{1}{(s-t)^{1+2 \varepsilon+\frac{2 n}{m}\left(\frac{1}{q}-\frac{1}{2}\right)}}\left(1+\frac{2^{j m}}{s-t}\right)^{-2 M}\|A(s, \cdot)\|_{q}^{2} d s t^{\beta} d t \\
& \cong \int_{0}^{1}\left(2^{k} t\right)^{2 \epsilon} \int_{2^{k} t}^{2^{k+1} t} \frac{1}{(s-t)^{1+2 \varepsilon+\frac{2 n}{m}\left(\frac{1}{q}-\frac{1}{2}\right)}}\left(1+\frac{2^{j m}}{s-t}\right)^{-2 M}\|A(s, \cdot)\|_{2}^{2} d s t^{\beta} d t \\
& =2^{2 k \epsilon} \int_{0}^{2^{k+1}} \int_{2^{-k-1} s}^{2^{-k} s} \frac{t^{\beta+2 \varepsilon}}{(s-t)^{\frac{2 n}{m}\left(\frac{1}{q}-\frac{1}{2}\right)+1+2 \varepsilon}}\left(1+\frac{2^{j m}}{s-t}\right)^{-2 M} d t\|A(s, \cdot)\|_{2}^{2} d s \\
& \cong 2^{2 k \epsilon} \int_{0}^{1}\left(2^{-k} s\right)^{\beta+2 \epsilon} \int_{2^{-k-1} s}^{2^{-k} s} \frac{1}{(s-t)^{\frac{2 n}{m}\left(\frac{1}{q}-\frac{1}{2}\right)+1+2 \varepsilon}}\left(\frac{2^{j m}}{s-t}\right)^{-2 M} d t\|A(s, \cdot)\|_{2}^{2} d s .
\end{aligned}
$$

We take $\epsilon \in\left(0, M-\frac{n}{m}\left(\frac{1}{q}-\frac{1}{2}\right)\right)$ so that the integral with respect to $t$ converges. Indeed, $M>\frac{n}{2 m} \geq \frac{n}{m}\left(\frac{1}{q}-\frac{1}{2}\right)$ and the calculation continues as follows:

$$
\begin{aligned}
& \cong 2^{2 k \epsilon} 2^{-k(\beta+2 \epsilon)} 2^{-2 M m j} 2^{-k} \int_{0}^{1} s^{2 M-\frac{2 n}{m}\left(\frac{1}{q}-\frac{1}{2}\right)-2 \epsilon}\|A(s, \cdot)\|_{2}^{2} s^{\beta+2 \epsilon} d s \\
& \lesssim 2^{-k(\beta+1)} 2^{-2 M m j} \int_{0}^{1}\|A(s, \cdot)\|_{2}^{2} s^{\beta} d s \\
& \leq 2^{-j n\left(\frac{2}{p}-1\right)} \lambda_{k, j}^{2}
\end{aligned}
$$

with $\lambda_{k, j} \cong 2^{\left(\frac{n}{2}\left(\frac{2}{p}-1\right)-M m\right) j} 2^{-\frac{k}{2}(\beta+1)}$, and we used $M>\frac{n}{2 m} \geq \frac{n}{m}\left(\frac{1}{q}-\frac{1}{2}\right)$.

If $j=0$ and $k \geq 1$, we do not use the decay but rather the fact that $(t-s) K(t, s)$ is uniformly bounded on $L^{2}\left(\mathbb{R}^{n}\right)$. Then we can repeat the above calculation literally taking $q=2$ and $M=0$.

If $k=0$ and $j=0$, using the boundedness of $T_{1}$ since $\beta>-1$,

$$
\int_{B(0,2)} \int_{0}^{2^{m}}\left|\left(T_{0,0} A\right)(t, y)\right|^{2} t^{\beta} d t d y \leq C \int_{B(0,1)} \int_{0}^{1}|A(s, x)|^{2} s^{\beta} d s d x .
$$

We conclude that $\lambda_{k, j} \cong 2^{\left(\frac{n}{2}\left(\frac{2}{p}-1\right)-M m\right) j} 2^{-\frac{k}{2}(\beta+1)}$ is summable for $\beta>-1$ and $M>\frac{n}{2 m}\left(\frac{2}{p}-\right.$ $1)$.

\section{Role of $L^{2}-L^{q}$ DECAY}

When $q \geq 2, L^{2}-L^{q}$ decay can be used to quantify $T^{p, 2}$ results for $p$ above 2 . Clearly the adjoint class to $S I O_{m, q, M}^{ \pm}$is $S I O_{m, q^{\prime}, M}^{\mp}$ with respect to the inner product

$$
\langle f, g\rangle=\int_{\mathbb{R}^{n}} \int_{0}^{\infty} f(t, y) \overline{g(t, y)} d t d y .
$$

It is easy to deduce from [13, Section 5] that for $p \in(1, \infty), m \in \mathbb{N}^{*}$ and $\beta \in \mathbb{R}$, we have $\left[T^{p, 2, m}\left(t^{\beta} d t d y\right)\right]^{\prime}=T^{p^{\prime}, 2, m}\left(t^{-\beta} d t d y\right)$, with duality given by $\langle f, g\rangle$, i.e.

$$
\|f\|_{T^{p, 2, m}\left(t^{\beta} d t d y\right)} \sim \sup _{\|g\|_{T^{p^{\prime}, 2, m}\left(t^{-\beta} d t d y\right)} \leq 1}|\langle f, g\rangle| .
$$


Thus, we obtain results for $2<p<\infty$ by dualizing Theorem 3.1 and Proposition 3.7 in the classes $S I O_{m, q, M}^{ \pm}$with $2 \leq q \leq \infty$ and $M>\frac{n}{2 m}$. In addition, the results for $p=\infty$ also hold.

Theorem 4.1. Let $T \in S I O_{m, q, M}^{-}$with $2 \leq q \leq \infty$ and $M>\frac{n}{2 m}$. Let $\beta>-1$.

(1) If $q \leq \frac{2 n}{m(1-\beta)}$ or equivalently $\frac{n}{2 m} \geq-\frac{\beta-1}{2}+\frac{n}{m}\left(\frac{1}{2}-\frac{1}{q}\right)$ then $T$ extends to a bounded operator on $T^{p, 2, m}\left(t^{\beta} d t d y\right)$ when $2 \leq p<p_{c}^{\prime}$, where

$$
p_{c}=\frac{2\left(\frac{n}{2 m}-\frac{n}{m}\left(\frac{1}{2}-\frac{1}{q}\right)\right)}{\frac{n}{2 m}-\frac{n}{m}\left(\frac{1}{2}-\frac{1}{q}\right)+\frac{1-\beta}{2}}=\frac{4 n}{2 n+m(1-\beta) q} .
$$

(2) If $q>\frac{2 n}{m(1-\beta)}$ or equivalently $-\frac{\beta-1}{2}+\frac{n}{m}\left(\frac{1}{2}-\frac{1}{q}\right)>\frac{n}{2 m}$ then $T$ extends to a bounded operator on $T^{p, 2, m}\left(t^{\beta} d t d y\right)$ when $2 \leq p \leq \infty$.

Proposition 4.2. Let $T \in S I O_{m, q, M}^{+}$with $2 \leq q \leq \infty$ and $M>\frac{n}{2 m}$. For all $\beta<1, T$ extends to a bounded operator on $T^{p, 2, m}\left(t^{\beta} d t d y\right)$ for $2 \leq p \leq \infty$.

It is enough to prove the result for $p=\infty$. The extension is done by taking $f \in$ $T^{\infty, 2, m}\left(t^{\beta} d t d y\right)$, truncating $f$ on $\left(0, k^{m}\right) \times B(0, k)$ and letting $k$ go to infinity.

Proof of Proposition 4.2. This is very similar to [8]. Pick a point $x_{0} \in \mathbb{R}^{n}$ and $r>0$. Let the sets $B_{j}$ and $C_{j}$ be defined as in the proof of Proposition 3.7. Set

$$
I^{2}=\int_{B\left(x_{0}, r\right)} \int_{0}^{r^{m}}|(T f)(t, y)|^{2} t^{\beta} d t d y .
$$

We want to show that $I^{2} \lesssim r^{n}\|f\|_{T^{\infty, 2, m}\left(t^{\beta} d t d y\right)}^{2}$. We set

$$
I_{j}^{2}=\int_{B\left(x_{0}, r\right)} \int_{0}^{r^{m}}\left|\left(T f_{j}\right)(t, y)\right|^{2} t^{\beta} d t d y
$$

where $f_{j}(s, x)=f(s, x) 1_{C_{j}}(s, x) 1_{\left(0, r^{m}\right]}(s)$ for $j \geq 0$. Thus by Minkowski inequality, $I \leq \sum I_{j}$. Since the proofs are scale and translation invariant, we assume $x_{0}=0$ and $r=1$ for simplicity. For $I_{0}$ we use again Theorem 2.2 which implies

$$
I_{0}^{2} \lesssim \int_{B(0,2)} \int_{0}^{2^{m}}|f(s, x)|^{2} s^{\beta} d s d x \lesssim\|f\|_{T^{\infty, 2, m}\left(t^{\beta} d t d y\right)}^{2} .
$$

Next, for $j \neq 0$, we proceed as in the proof of Proposition 3.7 by representing $T f_{j}(t, y)$ through a kernel (which is justified by the calculation below and Lemma 2.3 for the singular part) but using this time $L^{2}-L^{q}$ decay (after using Hölder inequality for the integral with respect to $y$ on $B(0,2))$ to obtain

$$
\begin{aligned}
I_{j}^{2} \lesssim & \sum_{k=1}^{\infty} \int_{0}^{1} \int_{2^{-k-1} t}^{2^{-k} t} \frac{2^{-k} t}{|t-s|^{\frac{2 n}{m}\left(\frac{1}{2}-\frac{1}{q}\right)+2}}\left(1+\frac{2^{j m}}{t-s}\right)^{-2 M}\left\|f_{j}(s, .)\right\|_{2}^{2} d s t^{\beta} d t \\
& +\int_{0}^{1} \int_{\frac{t}{2}}^{t} \frac{t^{\beta+2 \varepsilon}}{|t-s|^{\frac{2 n}{m}\left(\frac{1}{2}-\frac{1}{q}\right)+1+2 \varepsilon}}\left(1+\frac{2^{j m}}{t-s}\right)^{-2 M}\left\|f_{j}(s, .)\right\|_{2}^{2} d s d t
\end{aligned}
$$

Exchanging the order of integration, and using the fact that $t \sim t-s$ in the first part and that $t \sim s$ in the second, and $M>\frac{n}{m}\left(\frac{1}{2}-\frac{1}{q}\right)+\varepsilon$ for small enough $\varepsilon$, and $\beta<1$, we have the 
following.

$$
\begin{aligned}
& I_{j}^{2} \lesssim \sum_{k=1}^{\infty} 2^{-k} 2^{-2 j m M} \int_{0}^{2^{-k}} \int_{2^{k} s}^{2^{k+1} s} t^{\beta-1+2 M-\frac{2 n}{m}\left(\frac{1}{2}-\frac{1}{q}\right)}\left\|f_{j}(s, .)\right\|_{2}^{2} d t d s \\
& +\int_{0}^{1} \int_{s}^{2 s} \frac{t^{\beta+2 \varepsilon}}{|t-s|^{\frac{2 n}{m}\left(\frac{1}{2}-\frac{1}{q}\right)+1+2 \varepsilon}}\left(1+\frac{2^{j m}}{t-s}\right)^{-2 M}\left\|f_{j}(s, .)\right\|_{2}^{2} s^{\beta} d t d s \\
& \lesssim \sum_{k=1}^{\infty} 2^{-k} 2^{-2 j m M} \int_{0}^{2^{-k}}\left(2^{k} s\right)^{\beta}\left\|f_{j}(s, .)\right\|_{2}^{2} d s+2^{-2 j m M} \int_{0}^{1}\left\|f_{j}(s, .)\right\|_{L^{2}}^{2} s^{\beta} d s \\
& \lesssim 2^{-2 j m M} \int_{0}^{2^{j m}}\left\|f_{j}(s, .)\right\|_{2}^{2} s^{\beta} d s .
\end{aligned}
$$

We thus have

$$
I_{j}^{2} \lesssim 2^{-2 j m M} 2^{j n}\|f\|_{T^{\infty, 2, m}\left(t^{\beta} d t d y\right)}^{2}
$$

and the condition $M>\frac{n}{2 m}$ allows us to sum these estimates.

Proof of Theorem 4.1. The proof is almost entirely similar to the above one. Set $I_{j}$ as in the proof of Proposition 3.7. $I_{0}$ is estimated as before. When $j \geq 1$, the inner term in $I_{j}$ can be expressed using the kernel representation from $t$ to $+\infty$, which is split into $I_{j, k}$ on the dyadic intervals $\left(2^{k} t, 2^{k+1} t\right)$ for $k \in \mathbb{N}$, using Minkowski inequality. The $k=0$ term is estimated as was the term corresponding to $(t / 2, t)$. For $k \geq 1$, the $k$ th term is controlled by

$$
\int_{0}^{1} \int_{2^{k} t}^{2^{k+1} t} \frac{2^{k} t}{|t-s|^{\frac{2 n}{m}\left(\frac{1}{2}-\frac{1}{q}\right)+2}}\left(1+\frac{2^{j m}}{t-s}\right)^{-2 M}\left\|f_{j}(s, .)\right\|_{2}^{2} d s t^{\beta} d t .
$$

Exchanging order, we obtain the bound

$$
2^{-2 j m M} 2^{k\left(1-\beta+2 M-\frac{2 n}{m}\left(\frac{1}{2}-\frac{1}{q}\right)\right)} \int_{0}^{2^{k}}\left\|f_{j}(s, .)\right\|_{2}^{2} s^{\beta} d s .
$$

Note that the support of $f_{j}$ forces $s \leq 2^{(j+1) m}$ in the integral, which is bounded by $C 2^{j n}$. The series for $I_{j, k}$ is summable in $k$ under the condition in the statement and summable in $j$ if $M>\frac{n}{2 m}$.

\section{Maximal Regularity operators}

Let us come back to our original motivation which is to bound maximal regularity operators on tent spaces.

Definition 5.1. Let $1 \leq q \leq r \leq \infty$. A family of bounded linear operators $\left(T_{t}\right)_{t>0} \subset$ $B\left(L^{2}\left(\mathbb{R}^{n}\right)\right)$ is said to satisfy $L^{q}-L^{r}$ off-diagonal estimates of order $M$, with homogeneity $m$, if, for all Borel sets $E, F \subset \mathbb{R}^{n}$, all $t>0$, and all $f \in L^{2}\left(\mathbb{R}^{n}\right) \cap L^{q}\left(\mathbb{R}^{n}\right)$ :

$$
\left\|1_{F} T_{t} 1_{E} f\right\|_{r} \lesssim t^{-\frac{n}{m}\left(\frac{1}{q}-\frac{1}{r}\right)}\left(1+\frac{\operatorname{dist}(E, F)^{m}}{t}\right)^{-M}\left\|1_{E} f\right\|_{q} .
$$

With this definition we have the following simple fact.

Proposition 5.2. Let $1 \leq q \leq 2$ (resp. $2 \leq q \leq \infty)$ and assume that $\left(t L e^{-t L}\right)_{t \geq 0}$ satisfies $L^{q}-L^{2}$ (resp. $L^{2}-L^{q}$ ) off-diagonal estimates (of order $M$ ), with homogeneity $m$. Then $\mathcal{M}_{L} \in S I O_{m, q, M}^{+}$and $\mathcal{M}_{L^{*}}^{-} \in S I O_{m, q^{\prime}, M}^{-}$. 
Indeed, the operator-valued kernel $L e^{-|t-s| L}$ has $L^{q}-L^{2}$ (resp. $L^{2}-L^{q}$ ) decay (of order $M)$, with homogeneity $m$ so that it suffices to apply Definition 2.5

To illustrate our results so far, let us prove Proposition 1.5

Proof of Proposition 1.5. Let $L=-\Delta+V$ or $-\operatorname{div} A \nabla$ with real coefficients. Then, the kernel of the semigroup $\left(e^{-t L}\right)_{t>0}$ satisfies pointwise Gaussian estimate (see e.g. 24, Theorem $6.10]$ ), hence $L^{1}-L^{2}$ and $L^{2}-L^{\infty}$ off-diagonal estimates with homogeneity $m=2$ of order $\infty$. Therefore we have that $\mathcal{M}_{L} \in S I O_{2,1, \infty}^{+} \cap S I O_{2, \infty, \infty}^{+}$. We now apply the second case of Theorem 3.1 and Proposition 4.2 with $\beta=0$ to conclude that $T^{p, 2, m}(d t d y)$ boundedness of $\mathcal{M}_{L}$ holds for $\infty \geq p>\tilde{p}_{c}=\frac{n}{n+1}$.

Using the subordination formula, the Poisson semigroup associated with $\sqrt{L}$ satisfies $L^{1}-$ $L^{2}$ and $L^{2}-L^{\infty}$ off-diagonal estimates with homogeneity $m=1$ and order $\frac{n}{2}+1$. Thus $\mathcal{M}_{\sqrt{L}} \in S I O_{1,1, \frac{n}{2}+1}^{+} \cap S I O_{1, \infty, \frac{n}{2}+1}^{+}$. From $M=\frac{n}{2}+1$ and $m=1$, we have $p_{M}=\frac{n}{n+1}$. As $\beta=-1, m=1$ and $q=1, \frac{n}{2}<-\frac{\beta-1}{2}+\frac{n}{m}\left(\frac{1}{q}-\frac{1}{2}\right)=1+\frac{n}{2}$ and we are in the second case of Theorem 3.1. Applying this result and Proposition 4.2. we conclude that $T^{p, 2, m}\left(t^{-1} d t d y\right)$ boundedness of $\mathcal{M}_{\sqrt{L}}$ holds for $\infty \geq p>\sup \left(p_{M}, \tilde{p}_{c}\right)=\frac{n}{n+1}$.

As explained in the introduction, applications of our results require $M$ to be sufficiently large, namely $M>\frac{n}{2 m}$, whatever the value of $q$. Of course, with exponential decay, this is not a problem. Semigroups generated by elliptic operators of even order $m \geq 2$ have, in general, such an exponential off-diagonal decay. However, in the case of Poisson type semigroups, small polynomial decay is to be expected. This application suggests that the lower bound on $M$ should be kept as low as possible. Looking at the proof of Lemma 3.4 there seems to be unavoidable restrictions if we are only given $M$ without further information. However, the decay of the semigroup is usually computed from the decay of the resolvent and integration on a contour. This is the point of view we shall take.

We consider the following conditions on resolvent estimates for fixed $1 \leq q \leq r \leq \infty$.

1) There exists a bisectorial operator $\widetilde{L}$ of angle $\omega \in\left[0, \frac{\pi}{2}\right)$ having a bounded $H^{\infty}$ functional calculus on $L^{2}\left(\mathbb{R}^{n}\right)$ such that $L=|\widetilde{L}|\left(=\sqrt{\widetilde{L}^{2}}=\sqrt{L^{2}}\right)$, and for any $K \in \mathbb{N}$ and $\omega<\nu<\pi / 2$,

$$
\left\|1_{F}(1-z \widetilde{L})^{-1} 1_{E} f\right\|_{r} \leq c(K, \nu)|z|^{-\frac{n}{m}\left(\frac{1}{q}-\frac{1}{r}\right)}\left(1+\frac{\operatorname{dist}(E, F)^{m}}{|z|}\right)^{-K}\left\|1_{E} f\right\|_{q} .
$$

for all $f \in L^{2}\left(\mathbb{R}^{n}\right) \cap L^{q}\left(\mathbb{R}^{n}\right), E, F$ Borel subsets of $\mathbb{R}^{n}, z=e^{ \pm i \theta} t, t>0$ and $\left|\theta-\frac{\pi}{2}\right|<\frac{\pi}{2}-\nu$.

2) The operator $L^{2}$ is sectorial in $L^{2}\left(\mathbb{R}^{n}\right)$ of angle $2 \omega<\pi$ and for any $K \in \mathbb{N}$ and $\omega<\nu<\pi / 2$

$$
\left\|1_{F}\left(1-z L^{2}\right)^{-1} 1_{E} f\right\|_{r} \leq c(K, \nu)|z|^{-\frac{n}{2 m}\left(\frac{1}{q}-\frac{1}{r}\right)}\left(1+\frac{\operatorname{dist}(E, F)^{2 m}}{|z|}\right)^{-K}\left\|1_{E} f\right\|_{q}
$$

for all $f \in L^{2}\left(\mathbb{R}^{n}\right) \cap L^{q}\left(\mathbb{R}^{n}\right), E, F$ Borel subsets of $\mathbb{R}^{n}, z=e^{ \pm i \theta} t, t>0$ and $2 \nu<\theta \leq \pi$.

Operators of Dirac type satisfying (H1) with $m=1$ appear in [11, Proposition 5.2]. See also 7] and [19].

(H1) and (H2) are closely related and, in fact, (H1) implies (H2). Indeed, it follows from the resolvent formula

$$
\left(1-z^{2} \widetilde{L}^{2}\right)^{-1}=\frac{1}{2}(1-z \widetilde{L})^{-1}+\frac{1}{2}(1+z \widetilde{L})^{-1}
$$

for $z$ as in (H1). Remark that, in (H2), $2 w$ may be greater than or equal to $\pi / 2$, in which case $-L^{2}$ may not generate a semigroup.

Proposition 5.3. Let $L$ be a sectorial operator of angle $\omega<\pi / 2$ with an $H^{\infty}$ functional calculus on $L^{2}\left(\mathbb{R}^{n}\right)$. Assume that (H1) or (H2) is satisfied and fix $\omega<\nu<\pi / 2$. Then for any $0<\epsilon<R<\infty$ and any $\alpha \in \mathbb{C}$ with $\Re e \alpha \in[\epsilon, R],\left\|1_{F}(t L)^{\alpha} e^{-t L} 1_{E} f\right\|_{r}$ has bound

$$
c(\epsilon, R, q, r, \nu) e^{\nu|\Im m \alpha|} \cdot t^{-\frac{n}{m}\left(\frac{1}{q}-\frac{1}{r}\right)}\left(1+\frac{\operatorname{dist}(E, F)^{m}}{t}\right)^{-\Re e \alpha-\frac{n}{m}\left(\frac{1}{q}-\frac{1}{r}\right)}\left\|1_{E} f\right\|_{q} .
$$


A result in this spirit is in [16 for $q=r=2$.

Proof. It is enough to assume (H2). In this case, fix $\omega<\nu^{\prime}<\theta<\nu$, and let

$$
\phi_{t}(\lambda)=\left(t \lambda^{\frac{1}{2}}\right)^{\alpha} e^{-t \lambda^{\frac{1}{2}}}
$$

which is holomorphic and bounded for $|\arg \lambda|<\pi-2 \nu^{\prime}$. The Cauchy integral formula for sectorial operators implies that

$$
(t L)^{\alpha} e^{-t L}=\frac{1}{2 \pi i} \int_{\Gamma} \phi_{t}(\lambda)\left(1-\lambda^{-1} L^{2}\right)^{-1} \frac{d \lambda}{\lambda}
$$

holds with $\Gamma$ the oriented contour $\left\{|s| e^{i \operatorname{sign}(s) 2 \theta}: s \in \mathbb{R}\right\}$. We write $c_{\theta}=\Re e\left(e^{i \theta}\right)=\cos \theta>0$. Fix $f$ with $\left\|1_{E} f\right\|_{q}=1$. In the following, we write $a=\Re e \alpha, d=\operatorname{dist}(E, F), \gamma=\frac{n}{m}\left(\frac{1}{q}-\frac{1}{r}\right)$. Then (플 gives us

$$
\begin{aligned}
\left\|1_{F}(t L)^{\alpha} e^{-t L} 1_{E} f\right\|_{r} & \lesssim c(K) \int_{0}^{\infty} t^{a} s^{\frac{a}{2}} e^{\theta \Im m \alpha} e^{-c_{\theta} t s^{\frac{1}{2}}} s^{\frac{\gamma}{2}}\left(1+d^{2 m} s\right)^{-K} \frac{d s}{s} \\
& \cong c(K, \nu) e^{\nu|\Im m \alpha|} \int_{0}^{\infty} t^{a} s^{a} s^{\gamma} e^{-c_{\theta} t s}\left(1+d^{2 m} s^{2}\right)^{-K} \frac{d s}{s} \\
& \lesssim c(K, \nu) e^{\nu|\Im m \alpha|} t^{-\gamma} \int_{0}^{\infty} s^{a} s^{\gamma} e^{-c_{\theta} s}\left(1+\frac{d^{2 m} s^{2}}{t^{2}}\right)^{-K} \frac{d s}{s} \\
& \lesssim c(\epsilon, K, q, r, \nu) e^{\nu|\Im m \alpha|} t^{-\gamma}\left(1+\frac{d^{m}}{t}\right)^{-a-\gamma} .
\end{aligned}
$$

provided $2 K>R+\gamma$. We used the fact that $1 \leq 2(1+x)^{-1}$ when $x \leq 1$, and $x^{-1} \leq 2(1+x)^{-1}$ when $x \geq 1$. The parameter $\epsilon>0$ is only needed when $q=r$.

It is clear that similar results hold for fractional powers of sectorial operators. We shall not get into this here. Note also that an exponential decay in the resolvent estimates would not yield a better conclusion in general.

Definition 5.4. Let $L$ be a sectorial operator of type $\omega<\pi / 2$ and having a bounded holomorphic functional calculus on a Hilbert space $H$. For $\Re e \alpha>0$, we define the operator $\mathcal{M}_{\alpha}$ acting on $L^{2}\left(\mathbb{R}_{+}, d t ; D\left(L^{\alpha}\right)\right)$ by

$$
\mathcal{M}_{\alpha} f(t)=\int_{0}^{t}(t-s)^{\alpha-1} L^{\alpha} e^{-(t-s) L} f(s) d s .
$$

Clearly $\mathcal{M}_{1}=\mathcal{M}_{L}$.

Proposition 5.5. Let $\alpha \in\{z \in \mathbb{C} ; a \leq \Re e z \leq b\}$ for some $a, b \in \mathbb{R}_{+}$. Then $\mathcal{M}_{\alpha}$ extends boundedly to $L^{2}\left(\mathbb{R}_{+}, d t ; H\right)$, with a bound not exceeding ce ${ }^{\nu|\Im m \alpha|}$ for any $\omega<\nu<\pi / 2$, and some constant $c$ dependent on $a, b$.

Proof. Using operational calculus as in [3, which is possible since $L$ has bounded holomorphic functional calculus on $H$, it is enough to prove the same thing for $L=z I$ on $L^{2}\left(\mathbb{R}_{+}, d t ; \mathbb{C}\right)$ for $|\arg z|<\nu$. In this case, we use Schur's lemma for the complex-valued kernel $(t-$ $s)^{\alpha-1} z^{\alpha} e^{-(t-s) z} 1_{s<t}$. For $w=\Re e z,|z| \leq \frac{w}{\cos \nu}$, hence

$$
\begin{aligned}
\int_{0}^{t}\left|(t-s)^{\alpha-1} z^{\alpha} e^{-(t-s) z}\right| d s & \leq \frac{e^{\nu|\Im m \alpha|}}{(\cos \nu)^{\Re e \alpha}} \int_{0}^{t}\left|(t-s)^{\Re e \alpha-1} w^{\Re e \alpha} e^{-(t-s) w}\right| d s \\
& \leq \frac{\Gamma(\Re e \alpha) e^{\nu|\Im m \alpha|}}{(\cos \nu)^{\Re e \alpha}}
\end{aligned}
$$


and

$$
\begin{aligned}
\int_{s}^{\infty}\left|(t-s)^{\alpha-1} z^{\alpha} e^{-(t-s) z}\right| d t & \leq \frac{e^{\nu|\Im m \alpha|}}{(\cos \nu)^{\Re e \alpha}} \int_{s}^{\infty}\left|(t-s)^{\Re e \alpha-1} w^{\Re e \alpha} e^{-(t-s) w}\right| d t \\
& \leq \frac{\Gamma(\Re e \alpha) e^{\nu|\Im m \alpha|}}{(\cos \nu)^{\Re e \alpha}}
\end{aligned}
$$

with $\Gamma$ being the Euler Gamma function.

Corollary 5.6. Let $H=L^{2}\left(\mathbb{R}^{n}\right)$. If $1 \leq q \leq \infty$ and (H2) holds for $(q, 2)$ if $q \leq 2$ or $(2, q)$ if $q \geq 2$ then $\mathcal{M}_{\alpha} \in S I O_{m, q, M_{q}}^{+}$with $M_{q}=\Re e \alpha+\frac{n}{m}\left|\frac{1}{q}-\frac{1}{2}\right|$.

Observe that the order of decay becomes a function of $q$, hence the notation $M_{q} . M_{q}$ increases as $q$ moves away from 2: this is the interesting point for us. As mentioned in the introduction, $M_{2}=1$ is best possible for the Poisson semigroup of $-\Delta$, so it seems one cannot improve this conclusion.

Proof. The fact that $\mathcal{M}_{\alpha} \in S I O^{+}$is contained in Proposition 5.5. The decay of the kernel $(t-s)^{\alpha-1} L^{\alpha} e^{-(t-s) L}$ with $s<t$ is clear from Proposition 5.3 .

Corollary 5.7. Let $H=L^{2}\left(\mathbb{R}^{n}\right)$.

A] Assume (H2) holds for $(q, 2)$ with $q \leq 2$. Then $\mathcal{M}_{L}$ extends to a bounded operator on $T^{p, 2, m}\left(t^{\beta} d t d y\right)$ for $p_{L}<p<2$ with $p_{L}$ calculated as follows:

(1) If $\frac{n}{m q^{\prime}}<1$ and $\beta \leq-1, p_{L}=p_{M_{q}}$.

(2) If $\frac{n}{m q^{\prime}}<1$ and $-1<\beta<1, p_{L}=\inf \left(\tilde{p}_{c}, p_{c}\right)$.

(3) If $\frac{n}{m q^{\prime}} \geq 1$ then $\frac{1}{p_{L}}-\frac{1}{2}=\frac{m q^{\prime}}{n}\left(\frac{1}{\inf \left(p_{c}, 1\right)}-\frac{1}{2}\right)$.

B] Assume (H2) holds for $(2, q)$ with $q \geq 2$. Then $\mathcal{M}_{L}$ extends to a bounded operator on $T^{p, 2, m}\left(t^{\beta} d t d y\right)$ for $2<p<p_{L}$ with $p_{L}=\frac{2 n}{n-m q}$ if $m q \leq n$ and for $2<p \leq \infty$ if $m q>n$.

Note that the result for $p \geq 2$ does not depend on $\beta$. The exponents $p_{M_{q}}, \tilde{p}_{c}, p_{c}$ are explicitely defined in Theorem 3.1. The last two depend on $\beta$.

Proof. A] The condition $\frac{n}{m q^{\prime}}<1$ is equivalent to $M_{q}=1+\frac{n}{m}\left(\frac{1}{q}-\frac{1}{2}\right)>\frac{n}{2 m}$. Cases (1) and (2) thus follow from Theorem 3.1. In the third case, Theorem 3.1 does not apply to $\mathcal{M}_{L}$ but to $\mathcal{M}_{\alpha}$ for any $\alpha$ with $\Re e \alpha>\alpha_{1}$ and $\alpha_{1}+\frac{n}{m}\left(\frac{1}{q}-\frac{1}{2}\right)=\frac{n}{2 m}$ which implies that $\mathcal{M}_{\alpha}$ is bounded for $\inf \left(p_{c}, 1\right)<p<2$. At the same time, $\mathcal{M}_{\alpha}$ is bounded for $p=2$ when $\Re e \alpha>0$. The third case follows by complex interpolation for the analytic family $\mathcal{M}_{\alpha}$ (since the growth in $\Im m \alpha$ is admissible) in tent spaces.

B] The condition $m q>n$ means $M_{q}=1+\frac{n}{m}\left(\frac{1}{2}-\frac{1}{q}\right)>\frac{n}{2 m}$. So we apply Proposition 4.2 to $\mathcal{M}_{L}$. If $m q \leq n$, then we apply this result not to $\mathcal{M}_{L}$ but to $\mathcal{M}_{\alpha}$ for $\Re e \alpha>\alpha_{1}$ and $\alpha_{1}+\frac{n}{m}\left(\frac{1}{2}-\frac{1}{q}\right)=\frac{n}{2 m}$ and the $p=2$ result for $\Re e \alpha>0$ and conclude by interpolation for analytic families again.

Proof of Propositions [1.6 and 1.7. Write $L=-\operatorname{div} A \nabla$. We have that $\left(e^{-t L}\right)_{t \geq 0}$ satisfies pointwise Gaussian estimates if $n=1,2$. Hence the conclusion of the first part of Proposition 1.5 applies. For $n \geq 3$, let $1 \leq p_{-}(L)<p_{+}(L) \leq \infty$ be the numbers introduced in [1] such that for $p_{-}(L)<q \leq r<p_{+}(L),\left(e^{-t L}\right)_{t \geq 0}$ satisfies $L^{q}-L^{r}$ off-diagonal estimates with homogeneity $m=2$. As the decay is Gaussian, the order is $\infty$. Moreover, $p_{-}(L)<\frac{2 n}{n+2}$, $p_{+}(L)>\frac{2 n}{n-2}$ and, by [17, this is sharp for this class of complex operators. Taking $q<\frac{2 n}{n+2}$, we use the second item in Corollary [5.7 A] when $n=3,4$ and the third one when $n \geq 5$ to get the lower bound on $p$. For the upper bound $p=\infty$ included, we use $\mathrm{B}]$.

Now for the semigroup associated to $\sqrt{L}$. When $n=1$ or 2 , we have the pointwise Poisson kernel estimate, hence $L^{1}-L^{2}$ and $L^{2}-L^{\infty}$ off-diagonal estimates with order $\frac{n}{2}+1$ and homogeneity $m=1$. Hence the conclusion of the second part in Proposition 1.5 applies since $m=1$ and $\beta=-1$. For $n \geq 3$, with the same numbers $p_{-}(L), p_{+}(L)$ as above, the resolvent estimate (H2) holds with $m=1$ and $p_{-}(L)<q \leq r<p_{+}(L)$. Taking $q<\frac{2 n}{n+2}$, we 
use the first item in Corollary [5.7. A] when $n=3,4$ and the third one when $n \geq 5$ to get the lower bound on $p$. For the upper bound, we use B] with $q>\frac{2 n}{n-2}$ and find $\infty$ included if $n=3,4$, and the proposed value if $n \geq 5$.

Corollary 5.8. Let $H=L^{2}\left(\mathbb{R}^{n}\right)$.

A] Assume (H2) holds for $(2, q)$ with $2 \leq q$. Then $\mathcal{M}_{L}^{-}$extends to a bounded operator on $T^{p, 2, m}\left(t^{\beta} d t d y\right)$ for $2<p<p_{L}$ with $p_{L}$ calculated as follows:

(1) If $\frac{n}{m q}<1$ and $\beta \geq 1, p_{L}=\infty$ (and boundedness holds at $\infty$ ).

(2) If $\frac{n}{m q}<1$ and $-1<\beta<1, p_{L}=\infty$ (and boundedness holds at $p=\infty$ ) if $p_{c}<1$ and $p_{L}=p_{c}^{\prime}$ if $p_{c} \geq 1$.

(3) If $\frac{n}{m q} \geq 1$ then $\frac{1}{p_{L}}-\frac{1}{2}=\frac{m q}{n}\left(-\frac{1}{2}\right)=-\frac{m q}{2 n}$.

B] Assume (H2) holds for $(q, 2)$ with $q \leq 2$. Then $\mathcal{M}_{L}^{-}$extends to a bounded operator on $T^{p, 2, m}\left(t^{\beta} d t d y\right)$ for $p_{L}<p<2$ with $p_{L}=\frac{2 n}{n+m q^{\prime}}$ if $m q^{\prime} \leq n$ and $p_{L}=p_{M_{q}}$ if $m q^{\prime}>n$.

This time, this follows from Proposition 3.7 and Theorem 4.1 where one finds the value of $p_{c}$, using the operators

$$
\mathcal{M}_{\alpha}^{-} f(t)=\int_{t}^{\infty}(s-t)^{\alpha-1} L^{\alpha} e^{-(s-t) L} f(s) d s
$$

and the interpolation procedure of Corollary 5.7. Details are left to the reader.

\section{REFERENCES}

[1] P. Auscher, On necessary and sufficient conditions for $L^{p}$ estimates of Riesz transforms associated to elliptic operators on $\mathbb{R}^{n}$ and related estimates. Mem. Amer. Math. Soc. 871 (2007).

[2] P. Auscher, Changement d'angle dans les espaces de tentes. C. R. Acad. Sci. Paris, Ser. I 349 (2011) 297-301.

[3] P. Auscher, A. Axelsson, Weighted maximal regularity estimates and solvability of elliptic systems I. Invent. Math., (2011) 184: 47-115.

[4] P. Auscher, A. Axelsson, Remarks on maximal regularity estimates. 2010 Progress in Nonlinear Differential Equations and Their Applications, Vol. 60, 45-55, 2011 Springer Basel AG.

[5] P. Auscher, S. Hofmann, M. Lacey, A. McIntosh, P. Tchamitchian, The solution of the Kato square root problem for second order elliptic operators on $\mathbb{R}^{n}$. Ann. of Math. 156(2) (2002) 633-654.

[6] P. Auscher, J.M. Martell, Weighted norm inequalities, off-diagonal estimates and elliptic operators. II. Off-diagonal estimates on spaces of homogeneous type. J. Evol. Equ. 7 (2007) 265-316.

[7] P. Auscher, A. McIntosh, E. Russ, Hardy spaces of differential forms on Riemannian manifolds. J. Geom. Anal. 18 (2008) 192-248.

[8] P. Auscher, S. Monniaux, P. Portal, The maximal regularity operator on tent spaces, To appear in Communications on Pure and Applied Analysis.

[9] P. Auscher, S. Monniaux, P. Portal, Non-autonomous Cauchy problems, in preparation.

[10] P. Auscher, J. van Neerven, P. Portal, Conical stochastic maximal $L^{p}$-regularity for $1 \leq p<\infty$. Submitted, arXiv: 1112.3196

[11] A. Axelsson, S. Keith, A. McIntosh, Quadratic estimates and functional calculi of perturbed Dirac operators. Invent. Math 163(3) (2006) 455-497.

[12] S. Blunck, P. Kunstmann, Calderón-Zygmund theory for non-integral operators and the $H^{\infty}$-functional calculus, Rev. Mat. Iberoamericana 19 (2003) 919-942.

[13] R. Coifman, Y. Meyer, E.M. Stein, Some new function spaces and their applications to harmonic analysis. J. Funct. Anal. 62 (1985) 304-335.

[14] C. Fefferman, E.M. Stein, $H^{p}$ spaces of several variables. Acta Math. 129 (1972) 137-193.

[15] B. Haak, P. Kunstmann, Weighted admissibility and wellposedness of linear systems in Banach spaces. SIAM J. Control Optim. 45 (2007) 2094-2118.

[16] S. Hofmann, J.-M. Martell, $L^{p}$ bounds for Riesz transforms and square roots associated to second order elliptic operators. Publ. Mat. 47 (2003), no. 2, 497-515.

[17] S. Hofmann, S. Mayboroda, A. McIntosh, Second order elliptic operators with complex bounded measurable coefficients in $L^{p}$ Sobolev and Hardy spaces, Ann. Sci. Ecole Norm. Sup., série 4, 44, fascicule 5 (2011).

[18] E. Harboure, J.-L. Torrea, B. Viviani, A vector-valued approach to tent spaces, J. Anal. Math. Vol. 56 (1991), 125-140.

[19] T. Hytönen, J. van Neerven, P. Portal, Conical square function estimates in UMD Banach spaces and applications to $H^{\infty}$-functional calculi. J. Analyse Math. 106 (2008) 317-351.

[20] H. Koch, D. Tataru, Well-posedness for the Navier-Stokes equations. Adv. Math. 157 (2001), 22-35. 
[21] P.C. Kunstmann, L. Weis, Maximal $L^{p}$ regularity for parabolic problems, Fourier multiplier theorems and $H^{\infty}$-functional calculus, in Functional Analytic Methods for Evolution Equations (Editors: M. Iannelli, R. Nagel, S. Piazzera). Lect. Notes in Math. 1855, Springer-Verlag (2004).

[22] B. Muckenhoupt, Hardy's inequality with weights. Studia Math. 44 (1972) 31-38.

[23] J. van Neerven, $\gamma$-radonifying operators-a survey. Proc. Centre Math. Appl. Austral. Nat. Univ. 44 (2010) 1-61.

[24] E. M. Ouhabaz, Analysis of heat equations on domains. London Mathematical Society Monographs Series, 31. Princeton University Press, Princeton, NJ, 2005.

[25] J. Prüss, G. Simonett, Maximal regularity for evolution equations in weighted $L_{p}$-spaces, Arch. Math. (Basel) 82 (2004), no. 5, 415-431.

[26] L. de Simon, Un'applicazione della theoria degli integrali singolari allo studio delle equazioni differenziali lineare astratte del primo ordine. Rend. Sem. Mat., Univ. Padova (1964) 205-223.

PASCAL Auscher

Univ. Paris-Sud, laboratoire de Mathématiques, UMR 8628, F-91405 ORSAY; CNRS, F-91405

ORSAY.

pascal.auscher@math.u-psud.fr

Christoph Kriegler

Laboratoire de Mathématiques (UMR 6620), Université Blaise-Pascal (Clermont-Ferrand 2), Campus des Cézeaux, 63177 Aubière Cedex

christoph.kriegler@math . univ-bpclermont.fr

Sylvie MonniauX

LATP-UMR 7353, FST Saint-Jérôme, Univ. Aix-Marseille, F-13397 Marseille Cédex 20.

sylvie.monniaux@univ-amu.fr

Pierre Portal

Permanent Address:

Université Lille 1, Laboratoire Paul Painlevé, F-59655 Villeneuve D’AscQ.

Current Address:

Australian National University, Mathematical Sciences Institute, John Dedman Building, Acton ACT 0200, Australia.

pierre.portal@math.univ-lille1.fr 